\title{
Quasi-parallel electron beams and their possible application in inferring the auroral arc's root in the magnetosphere
}

\author{
J. Liang ${ }^{1}$, F. Jiang ${ }^{2}$, E. Donovan ${ }^{1}$, E. Spanswick ${ }^{1}$, V. Angelopoulos ${ }^{2,3}$, and R. Strangeway ${ }^{2}$ \\ ${ }^{1}$ Dept. of Physics and Astronomy, Univ. of Calgary, Canada \\ ${ }^{2}$ Institute of Geophysics and Planetary Physics, UCLA, Canada \\ ${ }^{3}$ Earth and Space Sciences Department, UCLA, Canada
}

Correspondence to: J. Liang (liangj@ucalgary.ca)

Received: 1 March 2013 - Revised: 3 May 2013 - Accepted: 22 May 2013 - Published: 20 June 2013

\begin{abstract}
In this study we investigate the upgoing electron beams at the topside ionosphere and their counterpart feature, the bidirectional quasi-parallel electron beams (QPEB) in the equatorial magnetosphere, with highlight on their potential application in estimating the location of the arc's root $(\mathrm{AR})$ in the magnetotail central plasma sheet (CPS). We infer from FAST data that the upgoing electron beam is often found in the equatorward vicinity of the inverted-V arc. On the premise of such a scenario, we propose a method to estimate the location of the AR from available magnetospheric measurements by assuming that the tailward boundary of the QPEB demarcates the earthward boundary of the AR. We report two events with THEMIS observations of QPEBs in the magnetotail CPS, and demonstrate how to use the QPEB features, together with the magnetic signatures of the current circuit constituted by the QPEB and arc, to estimate the earthward boundary of the AR. We find that the estimated earthward boundary of AR is situated at the periphery of a quasi-dipolar magnetosphere characterized by a strong $B_{\mathrm{Z}}$ gradient. This finding is consistent with previously existing proposals on the possible AR location in the tail (e.g., Lui and Burrows, 1978; Sergeev et al., 2012).
\end{abstract}

Keywords. Ionosphere (auroral ionosphere) - Magnetospheric physics (current systems; magnetotail)

\section{Introduction}

The questions of where an auroral arc maps to the equatorial magnetosphere and how to practically determine an arc's root in the magnetosphere have long been challenging and largely unanswered issues in space physics research. An au- roral arc is predominantly excited by electron precipitation, which carries an upward field-aligned current (FAC) out of the ionosphere. It is now commonly recognized that the main component of the precipitation fluxes powering the arc stems from an "auroral acceleration region" typically $1-2 R_{\mathrm{E}}$ above Earth. This auroral acceleration region decouples the magnetospheric particle population and the particles that actually excite the arc, and thereby hinders efforts to trace the particle features of the arc from magnetospheric measurements. Furthermore, currently available magnetic field and particle instruments do not have the desirable deployment and resolution to directly measure the local FAC density or the losscone flux in the equatorial magnetosphere. Therefore, a direct and definitive way to locate the arc's root (AR) in the magnetosphere has remained extremely difficult thus far.

To circumvent the above dilemma, efforts have been made recently in different ways. One established technique is to use comprehensive in situ datasets to tune the empirical magnetic field models to improve the mapping (Kubyshkina et al., 2011; Sergeev et al., 2012). Using such a technique, Sergeev et al. (2012) mapped the prebreakup arc to the CPS, and concluded that the AR is situated at the innermost part of the thin current sheet, yet at the outer periphery of the quasi-dipolar magnetosphere. This paper presents another approach - namely using certain "companion phenomena" that are expected to exist in the vicinity of the arc - to indirectly infer the AR. The companion phenomena must be well observable in the magnetosphere. Recently, using FAST and THEMIS GBO data, Jiang et al. (2012) found that the preexisting arc is consistently located near the interface between the large-scale region-1 and region-2 FACs. On the other hand, using NOAA observations, Sergeev et al. (2012) found 
that the prebreakup arc tends to be roughly colocated with a narrow peak of high-energy $(>30 \mathrm{keV})$ electron precipitations, a so-called "energetic electron arc". However, since neither the region-1/region- 2 current boundary nor the losscone flux of high-energy electrons are directly measurable or readily inferable from available magnetospheric data, they may not serve as ideal companion phenomena to locate the AR.

In this study, we shall attempt to use the upgoing electron beam as a companion feature of the arc. The upgoing electron beam is accelerated by quasi-static parallel electric fields in the downward FAC region, which is often found to be adjacent to the upward auroral FAC region and acting as the "return current" of the latter (Marklund and Karlsson, 2001). The quasi-static potential structure in the downward FAC region resembles in many aspects its counterpart (i.e., inverted-V) in the upward FAC region, except that it reverses in polarity and differs in the current-voltage relationship (see Marklund, 2009, for a comprehensive review). Overwhelming evidence of upward-accelerated electron beams came from LEO satellite observations such as S3-3 (Gorney et al., 1985), DE-1 (Burch et al., 1983), Viking (Hultqvist et al., 1988), Freja (Boehm et al., 1995), and in particular, FAST (e.g., Carlson et al., 1998; Elphic et al., 1998, 2000). In many strong upgoing electron beam events investigated, their mean energies typically range from $\sim 100 \mathrm{eV}$ up to a few $\mathrm{keV}$. When the electron beams are accelerated out from both hemispherical ionospheres and enter the magnetosphere, they undergo beam-plasma interactions, which are conducive to a number of plasma waves acting to scatter the electron beam and broaden its pitch-angular distribution (e.g., Zhang et al., 1993; Abel et al., 2002a). Such wave-induced diffusion processes compete with the adiabatic shrinking of pitch angle due to the reduction of the magnetic field inside the magnetosphere. The electron beams then become largely a "trapped" population that bounce within the magnetosphere. Eventually, the electron beams appear on magnetosphere satellite measurements as collimated beams with finite beam width ( $\sim 10-15^{\circ}$ in the nightside inner magnetosphere as surveyed by Abel et al., 2002b) along directions both parallel and antiparallel to the ambient magnetic field. Such bidirectional, collimated electron beams were previously reported on the basis of data from magnetospheric satellites such as ATS6 (Lin et al., 1979), AMPTE/CCE (Klumpar et al., 1988; Klumpar; 1993), CRRES (Abel et al., 2002a, b), and Cluster (Marklund et al., 2004; Wright et al., 2008). The nomenclature used to describe such phenomena differs from author to author, but in this paper we will use the terminology "quasiparallel electron beam" (QPEB). This QPEB phenomenon is generally well discernible by existing in situ particle instruments, and may serve as a tracer of the downward FAC region in the magnetosphere.

A natural extension to the above notions of the QPEB lies in that a spatial boundary of the QPEB region might bear the implication of a transition from a downward FAC to an upward FAC region. In particular, a number of observations and theoretical proposals regarding the generation mechanism and current system of the arc invoked a scenario of a radially confined FAC pair, of which a downward FAC sheet is located adjacent to the upward FAC sheet (e.g., Rothwell et al., 1991; Galperin et al., 1992; Galperin and Bosqued, 1999; Rönnmark and Hamrin, 2000; Rönnmark, 1999, 2002; Haerendel, 2007). Such a scenario of a "doublesheet FAC loop" will be discussed and checked with FAST data in Sect. 2 of this paper. Based upon the above scenario, the border of the upward FAC region tied to the arc may be estimated from the periphery of the downward FAC region, which is practically identifiable by tracing the spatial shut-off of the QPEB feature from in situ measurements. Certainly, such estimation cannot be confirmative by itself, but may nevertheless provide useful clues on the AR location, given the awkward situation that a direct and unequivocal determination of AR has been unachievable thus far based upon currently available observations and techniques.

Both the arc and the upgoing electron beam were found in various places of the auroral oval, implying the complexity of the fine structures of auroral FACs, and the variability of the current closure schemes (see, e.g., discussions in Elphic et al., 1998). Correspondingly, QPEBs were also observed in various regions of the magnetosphere, e.g., in the inner magnetosphere and the central plasma sheet (CPS) (Klumpar et al., 1988; Klumpar, 1993; Abel et al., 2002a, b), in the highlatitude PSBL (Marklund et al., 2004; Wright et al., 2008), or even in the polar cap (Teste et al., 2007). Therefore, it is important to first clarify the region of observation and interest in this study: (1) we are only interested in the premidnight sector, (2) we are only interested in the equatorwardmost, mesoscale (width $\gg 1 \mathrm{~km}$ ) arc close to the equatorward border of the auroral oval, and (3) we are only interested in the inner CPS $(L<12)$ region. Our region delimitation and event selection criteria are designed to reinforce the likelihood that the QPEB and AR are located close to each other in the magnetosphere, and together form a radially confined current circuit, a scenario constituting the base of this study. Furthermore, we are only interested in quiet and substorm growth-phase intervals instead of active times.

In this paper we shall report THEMIS observations of the QPEBs, and attempt to use the QPEB observations, aided by the magnetic signatures of the current circuit constituted by the QPEB and the arc, to estimate the earthward boundary of the AR in the CPS. The paper is arranged as follows. In Sect. 2 we shall exemplify the latitudinally adjacent bands of upward-accelerated electron beams and downgoing inverted- $\mathrm{V}$ arc structures from FAST measurements, and discuss their potential implications in magnetospheric observations. In Sect. 3, we shall report two THEMIS events of QPEB observations, and demonstrate how they can be used to infer the earthward boundary of the AR. We also discuss the inferred AR location with respect to a transition region from quasi-dipole field to stretched current sheet topology. 
Section 4 summarizes our proposed technique and concludes the paper.

\section{FAST observations of double-sheet FACs and their implication on magnetospheric observation}

According to the closure geometry of the FACs, the auroral current system is topologically classified into type I and type II (Bostrom, 1964). The auroral arc due to its extended length in east-west direction and narrow width in northsouth direction, has been commonly regarded as embedded in a current system of type II, in which the upward and downward FACs are azimuthally extended as "sheets", yet close meridionally (e.g., Rönnmark, 1999, 2002; Haerendel, 2007). In the premidnight sector of this paper's interest, the type II current system features an upward FAC sheet farther from Earth (higher in latitudes), and a downward FAC sheet closer to Earth (lower in latitudes). This arc-related current circuit has a similar latitudinal geometry to the large-scale region-1/region- 2 currents but is more intense in current density and presumably more confined in latitudinal/radial extent. As a matter of fact, a number of existing proposals on the generation mechanism and current system of the arc predicted a double-sheet FAC configuration, with a downward FAC sheet located immediately earthward of the upward FAC sheet in the premidnight sector (e.g., Rothwell et al., 1991; Galperin et al., 1992; Galperin and Bosqued, 1999).

To check the scenario of the double-sheet FAC configuration associated with the arc, we have looked at FAST satellite data for three selected months (February 1997, September 1997, and February 2008) when the satellite regularly crossed the premidnight-evening auroral oval, and the satellite altitude was suitable for detecting upward-accelerated electrons (> $2000 \mathrm{~km}$; see Carlson et al., 1998). The FAST satellite (Pfaff et al., 2001) carries a fluxgate magnetometer and four electrostatic analyzers that measure particle fluxes of energies from a few $\mathrm{eV}$ to $\sim 30 \mathrm{keV}$ in various angular directions. We collect events with coexisting upgoing electron beam and inverted-V arc structures. Our survey criteria are as follows. In accordance with the results in Jiang et al. (2012), we identify the "arc" from the inverted-V type of precipitation with characteristic energy ranging from hundreds of $\mathrm{eV}$ up to a few $\mathrm{keV}$. We set a threshold for the peak precipitating energy flux (mapped to $\sim 100 \mathrm{~km}$ ionosphere) associated with the inverted-V arc to exceed $1 \mathrm{erg} \mathrm{cm}^{-2} \mathrm{~s}^{-1}-$ which is the threshold for visible arcs. Consistent with our research interest in the equatorward-most arc, we stipulate the criteria that the inverted- $\mathrm{V}$ arc must be an isolated structure located near the equatorward border of the electron auroral oval and/or at the poleward edge of diffuse electron precipitation. There might be some other prominent inverted-V structures poleward of the arc of interest, but none further equatorward of this arc. The upgoing electron beam of our interest must extend to energies above $100 \mathrm{eV}$ (we shall discuss such en- ergy threshold later in this section), with differential energy flux on the order of $10^{9} \mathrm{eV} \mathrm{cm}^{-2} \mathrm{~s}^{-1} \mathrm{sr}^{-1} \mathrm{eV}^{-1}$ or higher.

Figure 1 shows four event examples in our surveyed months. The 26 September 1997 event was previously investigated by Lessard et al. (2007) with conjugate optical auroral observations, though the upgoing electron beam and the FAC geometry are not included in their research interest. The events occur in different hemispheres, but the plot format in each subfigure is identical: the first three panels show the electron energy flux spectrograms for downgoing $\left(0-30^{\circ}\right.$ pitch angle in the Northern Hemisphere and $150-180^{\circ}$ in the Southern Hemisphere), quasi-perpendicular $\left(60-120^{\circ}\right.$ pitch angle), and upgoing (150-180 pitch angle in the Northern Hemisphere and $0-30^{\circ}$ in the Southern Hemisphere) directions. In all events, distinct inverted-V structures near the equatorward edge of the auroral oval are identified from the electron flux spectrogram in the downgoing and perpendicular directions. Immediately equatorward of the inverted- $\mathrm{V}$ structures, upgoing electron beam structures with energies extending above $100 \mathrm{eV}$ are seen in all events. Panel 4 shows the ion energy flux spectrogram for the downgoing direction. Comparing the relative latitudes of the upgoing electron beam, the inverted-V structure, and the region of intense precipitations of $>1 \mathrm{keV}$ protons, one may notice that the inverted-V is located distinctly poleward of the intense proton precipitations (Lessard et al., 2007; Jiang et al., 2012). The upgoing electron beam is located between the region of peak proton precipitation and that of the inverted- $\mathrm{V}$ arc, and is partly colocated with, or at the poleward end of, the intense proton precipitation. Panels 5 and 6 display the magnetic field deviations on FAST, and the inferred FACs from them, respectively. Not surprisingly, the upgoing electron beam and the inverted- $\mathrm{V}$ structure are associated with strong downward FAC and upward FAC, respectively. The downward FAC is located equatorward of, yet latitudinally adjacent to, the upward FAC, forming a double-sheet FAC geometry as theoretically expected. It is beyond this paper's interest to pursue fine details of each event, but we shall mention a few subtleties and complications in those events, some of which will be readdressed in our later analyses: (a) in the 21 September 1997 event there were a few upgoing electron beam structures with energies $<100 \mathrm{eV}$ at lower latitudes; (b) in the 26 September 1997 event, there was a glitch of the FAST ESA instrument during the time of 06:13:36-06:13:38 Utc, which overlaps with the occurrence interval of the upgoing electron beam, and prevents us from knowing the equatorward boundary and the actual width and of the electron beam - the FAC density shown in the bottom panel indeed hints that the upgoing electron beam (downward FAC peak) likely has a wider latitudinal scale masked by the glitch. Nevertheless, the poleward boundary of the upgoing electron beam and its relative geometry with respect to the inverted- $\mathrm{V}$ arc, which are the key observations of our interest, are unambiguous to see. (c) In the 1 February 1997 event, strong upgoing electron beams existed both equatorward and poleward 

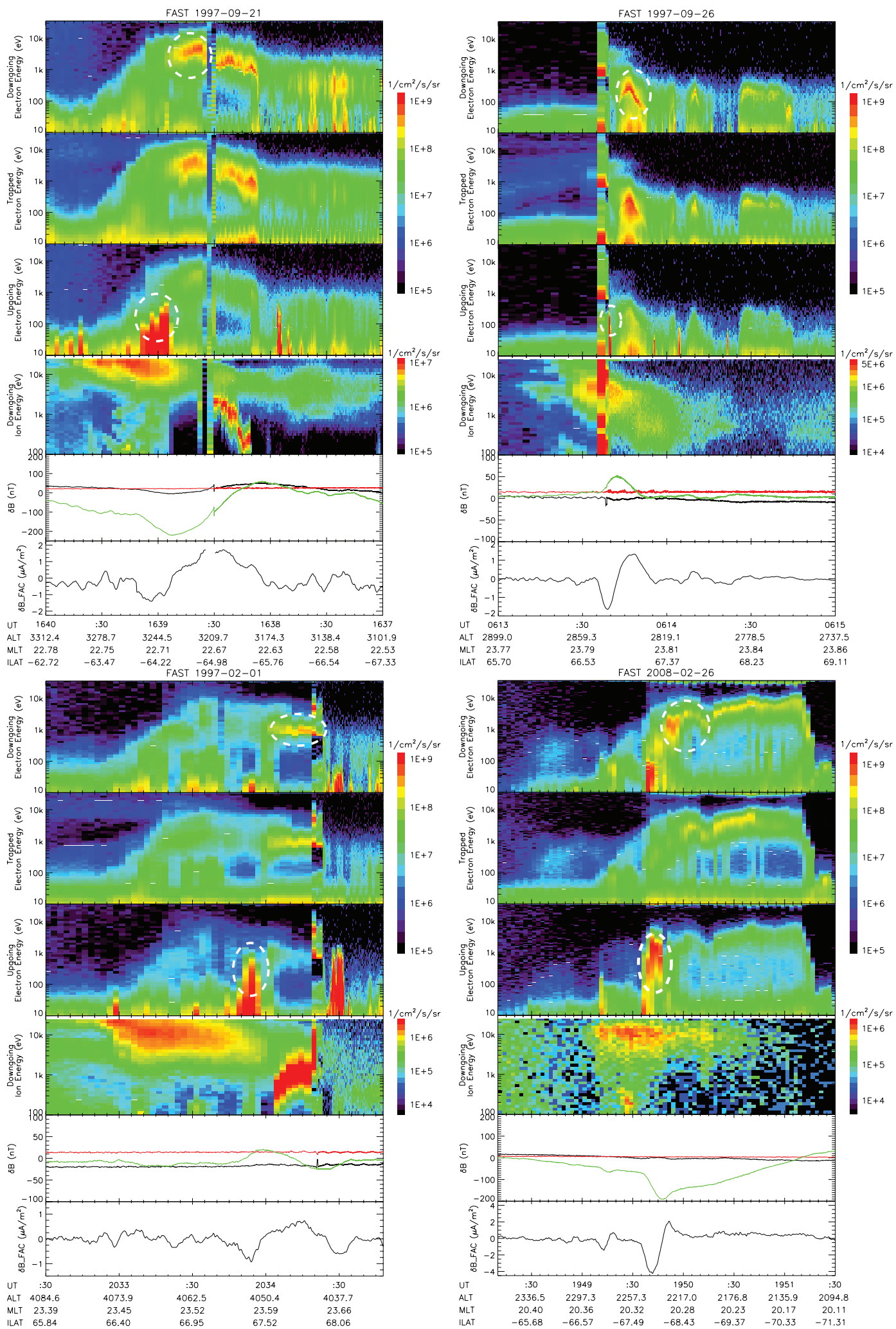

Fig. 1. FAST observations for four events. The plot format is identical for each event and subfigure. The top three panels show the electron energy flux spectrogram in downgoing, perpendicular, and upgoing directions. Two dashed circles serve to highlight the key structures of interest: the inverted-V arc and upgoing electron beam. The 4th panel shows the ion energy flux spectrogram in downgoing direction. the 5th panel show the deviations of magnetic fields: red, blue, and green curves denote the perturbations along the ambient B-field direction, along north-south direction (positive southward), and along east-west direction (positive eastward), respectively. The bottom panel shows the spin-smoothed FAC derived from the magnetic field deviations; negative (positive) values indicate downward (upward) FAC. 


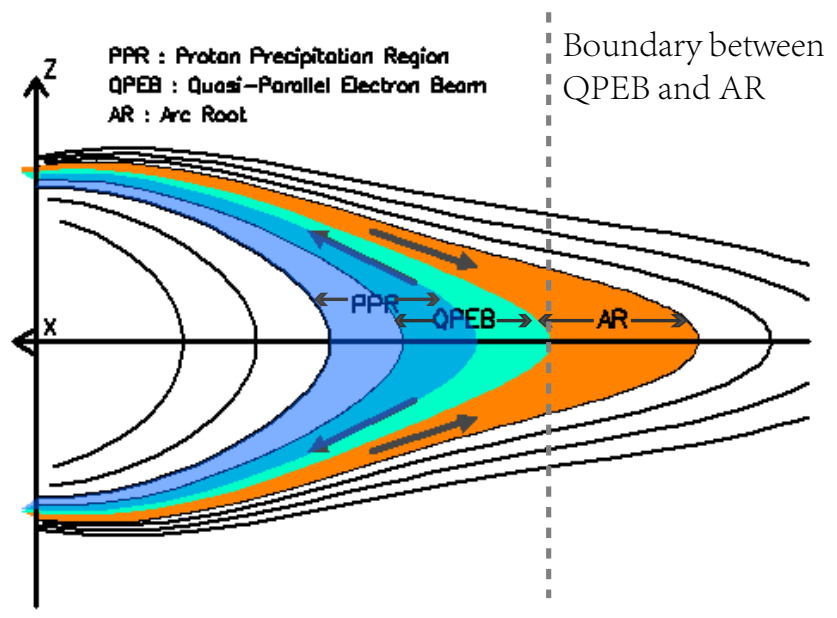

Fig. 2. Schematic diagram showing the magnetospheric regions of our interest, including the proton precipitation region, the QPEB region, and the AR. Solid arrow indicates the sense of FAC in each region. A vertical line highlights the core observational interest in this study: a boundary between the upgoing electron beam and arc in the ionosphere, or equivalently a boundary between the QPEB and $\mathrm{AR}$ in the equatorial CPS.

of the inverted-V arc. Accordingly, the upward FAC is sandwiched between two downward FAC sheets, forming a threesheet FAC configuration (Dubyagin et al., 2003). (d) In the 26 February 2008 event, the downgoing electron structure (around 19:48:50 UTC) that carries the peak upward FAC was not a classical isotropic inverted-V. Subject to one's criterion and nomenclature, this structure may be classified as a "field-aligned electron burst", though this electron burst structure is still mono-energetic, and situated at the equatorward edge of an ambient weaker inverted-V structure. We also note that the energy range of the upgoing electron beam is exceptionally high in this event, extending up to $\sim 3 \mathrm{keV}$. The above observations suggest that the generation mechanisms of the arc and the upgoing electron beam might be somehow special in this particular event. Nevertheless, the double-sheet FAC configuration, and its relative geometry with respect to the proton precipitation region, still fully comply with the common scenario revealed in other events.

So far as we have surveyed three months' FAST data, we are able to identify a total of 25 events that are similar to those presented in Fig. 1. All events share the same features - namely (a) a strong upgoing electron beam is found in the equatorward vicinity of an inverted- $\mathrm{V}$ arc; (b) the upgoing electron beam is partly colocated with, or at the poleward end of, the intense ion precipitation region, while the arc is located further poleward, at the negative slope of the ion precipitation versus increasing latitude. The scenario of doublesheet FAC geometry associated with the arc is thus repeatedly discerned. Such double-sheet FAC pattern is also discerned in a number of conjunctive events of CHAMP measurements of the most equatorward arcs (D. Knudsen, pri- vate communication, 2012). The scenario is also compatible with the results of Ohtani et al. (2010) and Jiang et al. (2012) that an equatorward-most arc is often located immediately poleward of a downward FAC region in the premidnight sector. Our survey is still undergoing and is the subject of a future publication. Admittedly, our event pool so far may not be sufficient to assert a statistical significance, and it is certainly true that FAC pattern surrounding the arc may exhibit a variety of fine structures (Y. Nishimura, private communication, 2012). Nevertheless, based upon our preliminary survey, we feel confident to suggest that the "double-sheet FAC" scenario represents one common type of current closure geometry associated with an auroral arc. Most importantly, the events we have surveyed unveil a consistent latitudinal sequence of three auroral phenomena of our research interest: the proton precipitation, the upgoing electron beam, and the auroral arc. Such a latitudinal sequence of the three auroral phenomena, when mapped to the magnetosphere, suggests a radial structuring of their respective counterparts in the CPS. In Fig. 2 we present a schematic diagram showing the magnetospheric regions of our interest. We shall discuss the expected signature and traceability of those regions on the basis of available observational datasets from ground and in situ instruments:

1. The peak region of proton precipitation. This region will manifest itself as the proton aurora band in the ionosphere. The proton precipitation flux is a convolution of the ambient trapped flux and the pitch-angle scattering rate. For CPS thermal ions, the most recognized mechanism of such pitch-angle scattering is contributed by the nonadiabatic, stochastic ion motion at the neutral sheet (Sergeev et al., 1983); the resulting scattering rate depends on the particle gyroradius (and thus energy) and on the radius of curvature of the magnetic field near the neutral sheet. Based upon a statistical study of growth-phase CPS pressure and magnetic fields, $\mathrm{Za}$ haria and Wang (2011) estimated the magnetic fieldline curvature at the neutral sheet by assuming magnetostatic force balance. Their results suggest that in the premidnight CPS at $L \sim 10$, which is the region of our interest in this study, a condition of strong pitchangle scattering is generally met for $>5 \mathrm{keV}$ protons (C.-P. Wang, private communication, 2012). In this regard, the parallel or antiparallel flux of ions with energies above a few $\mathrm{keV}$, measured in angular bins close to the loss cone, might be used as a rough proxy to gauge the precipitation flux of CPS protons and in turn the proton auroral intensity.

2. The QPEB region, which constitutes the core interest of this study. This QPEB region is partly colocated with and/or at the tailward end of the peak proton precipitation region. General features and mechanisms of the QPEB have been introduced in the previous section. When the upgoing electron beams are fully accelerated 
out of the parallel potential region and enter the magnetosphere, their energy spectra will tend to be "band limited", with the core energy bearing the implication of the total parallel potential drop. As we shall exemplify later via THEMIS observations, the QPEB often appears as a readily distinguishable feature in magnetospheric in situ observations. A proper determination of the tailward boundary of the QPEB region constitutes the key procedure of this study. Admittedly, the in situ measurement always involves a compound mixture of temporal and spatial variations, and the passage of a satellite in the magnetosphere is constantly entangled with motions of magnetospheric regions. Therefore, throughout the paper, when we say a satellite "moves" into a certain region, we always imply a relative motion. However, under the conditions of quasi-stationarity and slow convection of our interest, no matter what the relative motion is between the probe and the magnetospheric region, a "crossing of border" between neighboring regions remains meaningful, though the exact dimension of each region is ambiguous. Furthermore, multiprobe analysis using closely spaced satellites can further help relieve the temporal/spatial ambiguity. As we shall demonstrate in the following THEMIS events, with the above procedures a spatial boundary of the QPEB region can be practically determined with certain confidence.

3. The AR in the CPS. As we have discussed in the previous section, there is no unambiguous way to directly measure the AR location in the magnetosphere. On magnetic field lines tied to the arc, upgoing ionospheric electrons are expected to be suppressed due to the presence of upward E fields in the auroral acceleration region. In this regard, the outer boundary of the QPEB region thereby demarcates the possible radial range of $\mathrm{AR}$, in that the AR cannot extend further earthward of such a boundary into the QPEB region. Therefore, we consider the outer boundary of QPEB as a potential indicator of the inner boundary of the AR. Inferred from our preliminary survey, the "center" of the arc, which is defined by a peak of the downgoing electron energy flux, is generally within $\sim 0.1-0.4^{\circ}$ ILAT of the poleward boundary of the upgoing electron beam. Using a radial mapping factor $\sim 200$ at $L \sim 10$ inferred from empirical magnetic models, the center of the AR region would then be located slightly tailward of the QPEB region, separated by a distance of $\sim 0.3-1.2 R_{\mathrm{E}}$. Therefore, tracing the outer boundary of QPEB may yield meaningful inference on the AR location.

Our final note in this section is to address two known exceptions/complications, realized from our survey and the existing literature, to the scenario shown in Fig. 2, and our methods to overcome the ambiguities brought by those complica- tions. Note that we have purposefully chosen example events with those complications in Fig. 1.

1. In a number of FAST events we surveyed, there might be one or more upgoing electron structures, with energies of only a few tens of eV, randomly appearing at latitudes equatorward of the higher-energy electron beam of our interest. The 21 September 1997 event in Fig. 1 gives such an example. Note that those low-energy electron beams at lower latitudes are located on a shallow negative slope of the azimuthal magnetic field; they are possibly related to some weak downward FACs characteristic of the ambient duskside region-2 current, but have no definite relationship to the arc. The upgoing electron beam of our interest is higher in energy range, and is associated with a substantially stronger downward FAC that are more likely arc related. We impose a $\sim 100 \mathrm{eV}$ threshold for both the upgoing electron beams and the QPEB to be considered in this study, which is explained as follows. The energy threshold of the upgoing electron beam or QPEB points to the requirement of the potential drop and in turn the downward FAC density. The electron current density produced by a parallel potential structure with a potential drop $\Phi_{/ /}$can be estimated as $j_{/ /} \approx N_{\mathrm{e}} \cdot \sqrt{2 e \Phi_{/ /} / m_{\mathrm{e}}}$, which gives (Temerin and Carlson, 1998)

$\Phi_{/ /} \approx m_{\mathrm{e}} j_{/ /}^{2} / 2 N_{\mathrm{e}}^{2} e^{3}$.

Note that, while the electrons are the main current carrier, a charge neutrality has to be maintained along the flux tube to a large extent. Hence the electron density $N_{\mathrm{e}}$ cannot exceed the ion density along the flux tube. Assuming $N_{\mathrm{i}} \sim 1 \mathrm{~cm}^{-3}$ on a flux tube threading the CPS, to achieve a $1 \mu \mathrm{Am}^{-2}$ FAC density, $\Phi_{/ /}=110.8 \mathrm{~V}$ is required. When complications such as ion mobility are considered in the theory (Temerin and Carlson, 1998; Cran-McGreehin and Wright, 2005), the required potential drop can be even larger than that stipulated by Eq. (1). As a matter of fact Temerin and Carlson (1998) and Cran-McGreehin and Wright (2005) both concluded that, to maintain a downward $j_{/ /}>1 \mu \mathrm{Am}^{-2}$, a potential drop of the order of several hundred $\mathrm{V}$ up to a few $\mathrm{kV}$ is required. For our research interest on the return current associated with a visible auroral arc, we argue that our imposed energy threshold for the QPEB is justifiable.

2. In our survey we occasionally see a few events with upgoing electron beams on both sides of an inverted- $\mathrm{V}$ arc - e.g., the 1 February 1997 event shown in Fig. 1. In such a case, the arc is sandwiched between two sheets of downward FACs, one equatorward and the other poleward of the arc. Another event example of three-sheet FAC structure centered around an arc was investigated 
by Dubyagin et al. (2003), though in their event the FAST altitude is too low $(\sim 400 \mathrm{~km})$ to detect the upgoing electron beam feature. Therefore, when a QPEB region is observed in the CPS, there might be an ambiguity whether the QPEB is earthward or tailward of an AR. Such ambiguity is however manageable: (a) the arc is consistently located poleward of the peak band of proton auroras (Samson et al., 1992; Jiang et al., 2012; Donovan et al., 2012). Inferred from our survey, the upgoing electron beams equatorward of the arc tend to be partly colocated with, or at the poleward end of, the intense proton precipitation region, while for the occasional existence of upgoing electron beams poleward of the arc, those poleward electron beams are relatively far away from the peak proton precipitation region, and are at a place of much-diminished proton fluxes (see 1 February 1997 event for an example). Therefore, the relative geometry between the QPEB and the peak region of parallel/antiparallel fluxes of $>5 \mathrm{keV}$ protons may provide crucial evidence to distinguish whether the QPEB is located tailward or earthward of an AR. Such procedure is to be exemplified in Event 1 later in Sect. 3. (b) Though our interest is limited to quiet and growthphase intervals, if a substorm onset ensues and the arc eventually breaks up, which is the case in Event 2 to be shown in Sect. 3, the propagation sequence of the substorm disturbance can also help to resolve the ambiguity of the AR location.

\section{THEMIS and ground optical observations: QPEB and $\mathbf{A R}$}

In this section we shall use THEMIS in situ observations, together with conjugate ground optical observations, to demonstrate the above-proposed technique and procedure. The main THEMIS instruments used in this study include the fluxgate magnetometer (FGM, see Auster et al., 2008), and the electrostatic analyzer (ESA, see McFadden et al., 2008). The FGM instrument measures the in situ magnetic field. The ESA instrument measures the flux of thermal particles over an energy range from $5 \mathrm{eV}$ to $25 \mathrm{keV}$ for ions, and $6 \mathrm{eV}$ to $28 \mathrm{keV}$ for electrons. Full angular resolution ESA data are utilized, containing 88 angular bins per spin $(3 \mathrm{~s})$. We also use the FilterBank (FBK) dataset (Cully et al., 2008) of the electric field instrument (EFI, see Bonnell et al., 2008) and the search coil magnetometer (SCM, see Roux et al., 2008) to investigate the wave activity. For ground optical auroral observations, we shall mainly use the multispectrum imager (MSI) and the meridian scanning photometer (MSP) at Fort Smith (FSMI), Gillam (GILL), and Rankin Inlet (RANK) stations of the Northern Solar Terrestrial Array (NORSTAR) mission.

The THEMIS satellite orbit geometry during the two events presented here is given in Fig. 3. Both satellite pro- (a)

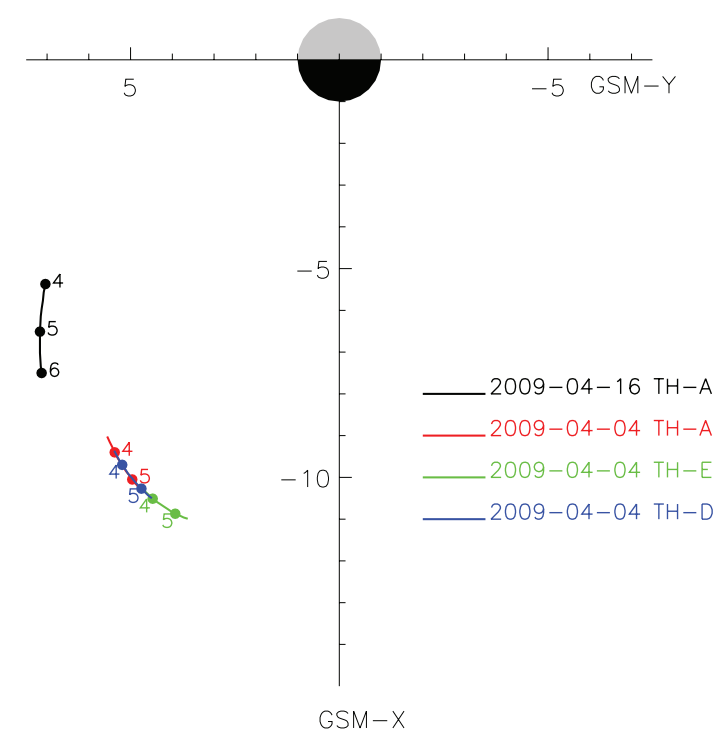

(b)

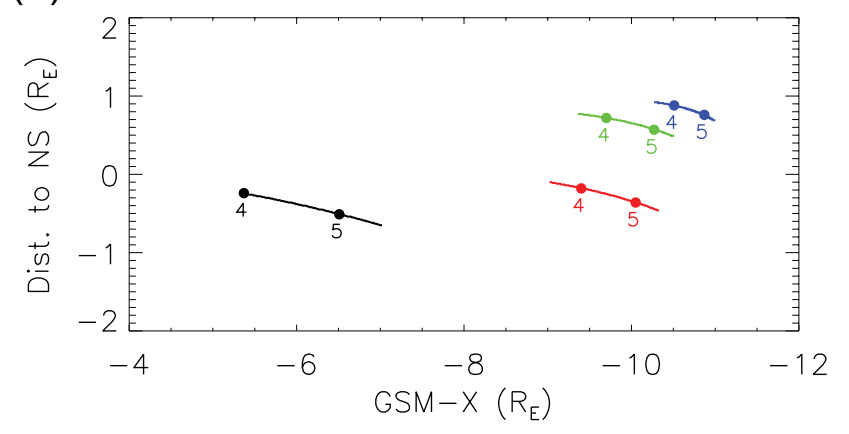

Fig. 3. (a) THEMIS satellite orbits on GSM-XY plane during the two event intervals on 16 April 2009 and 4 April 2009. The color codes for the date and probe are labeled in the plot. (b) The distance of the probes to Tsyganenko model neutral sheet versus GSM-X during the two event intervals.

jections on the GSM-XY plane (Fig. 3a) and their distances to the Tsyganenko model neutral sheet (Fig. 3b) are shown. TH-A is used in Event 1, and TH-A/D/E are used in Event 2. TH-A was south of the neutral sheet in both events, while in Event 2 TH-D/E were in the northern CPS and farther off the neutral sheet than TH-A. Note that the above-shown geometry of the satellites with respect to the neutral sheet is qualitatively consistent with that inferred from actual observations of the $B_{\mathrm{x}}$ and plasma $\beta$ to be presented later in this section.

Before we delve into the data analyses we shall first explain one computational note on how we practically define "radial" and "azimuthal" directions locally in the CPS, when we refer to those components of B fields or flows. In the existing literature and common understanding, depending upon the region of interest, a radial (azimuthal) direction is often defined as either $\hat{\boldsymbol{e}}_{\mathrm{r}}\left(\hat{\boldsymbol{e}}_{\varphi}\right)$ in a dipole-cylindrical coordinate or 


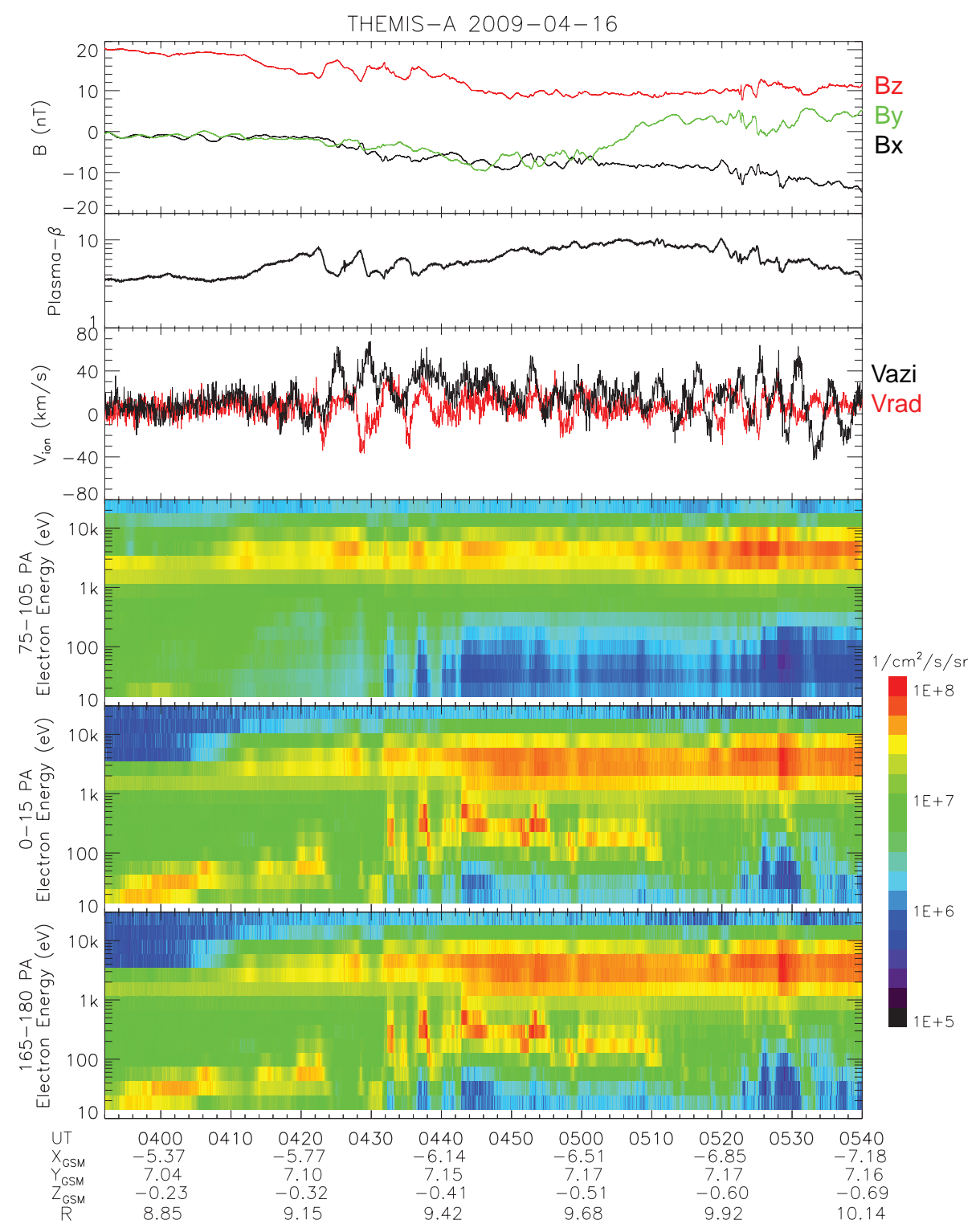

Fig. 4. TH-A observations on 16 April 2009. From top to bottom panels are the magnetic fields in GSM components, the plasma $\beta$, the azimuthal (positive duskward) and radial (positive earthward) ion flows, and the electron energy flux spectrograms in perpendicular $(\alpha \sim 75-$ $105^{\circ}$ ), parallel $\left(\alpha \sim 0-15^{\circ}\right)$, and antiparallel $\left(\alpha \sim 165-180^{\circ}\right)$ directions.

GSM-X (GSM-Y) direction in a highly stretched tail. Since the region of our interest potentially covers a transition from quasi-dipole to stretched field line topology, a more flexible definition of the radial/azimuthal directions is desirable. In this study the radial direction is determined from the local $\nabla V$ direction projected on the GSM equator, in which $V$ is the flux tube volume per unit flux calculated from Tsyganenko model (T02 version), and the azimuthal direction is defined as along $\nabla V \times \hat{z}$. Though empirical in nature, the Tsyganenko models can basically reproduce the expected transition of $\nabla V$ from $\hat{\boldsymbol{e}}_{\mathrm{r}}$-directed closer to Earth to GSM$\mathrm{X}$-aligned farther in the tail (see, e.g., Fig. 9 in Gkioulidou et al., 2011). We have tested different empirical models with various model parameters, and verified that the key features of the azimuthal B fields and flows we are to present are insensitive to the empirical model chosen.

\subsection{April 2009 event}

On 16 April 2009, 04:00-06:00 UTC, TH-A was radially outbound in the premidnight magnetosphere. Inferred from the solar wind data on OMNIWEB, the IMF $B_{\mathrm{Z}}$ had been dominantly negative for many hours, and steadily decreased from $\sim-3$ to $-6 \mathrm{nT}$ during the interval 04:00-06:00 UTC, implying that the magnetotail was fairly stretched. The event occurred during an interval of relatively quiet geomagnetic 


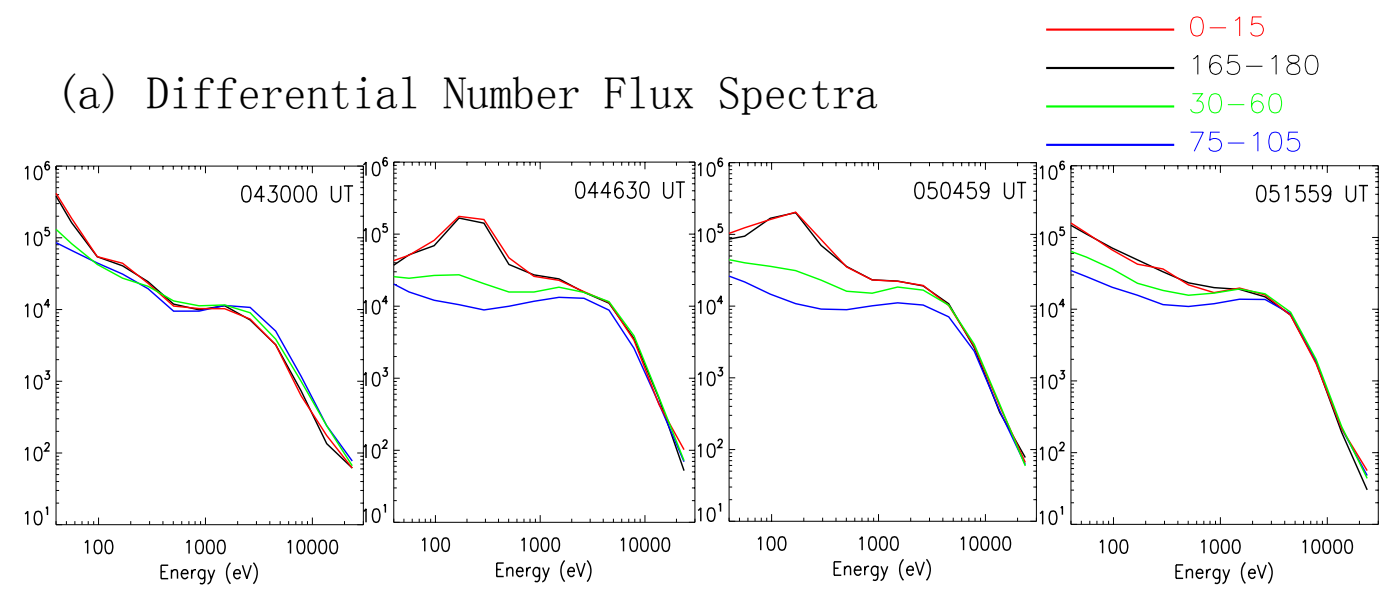

(b) Pitch-Angle spectrogram of QPEB and Wave data

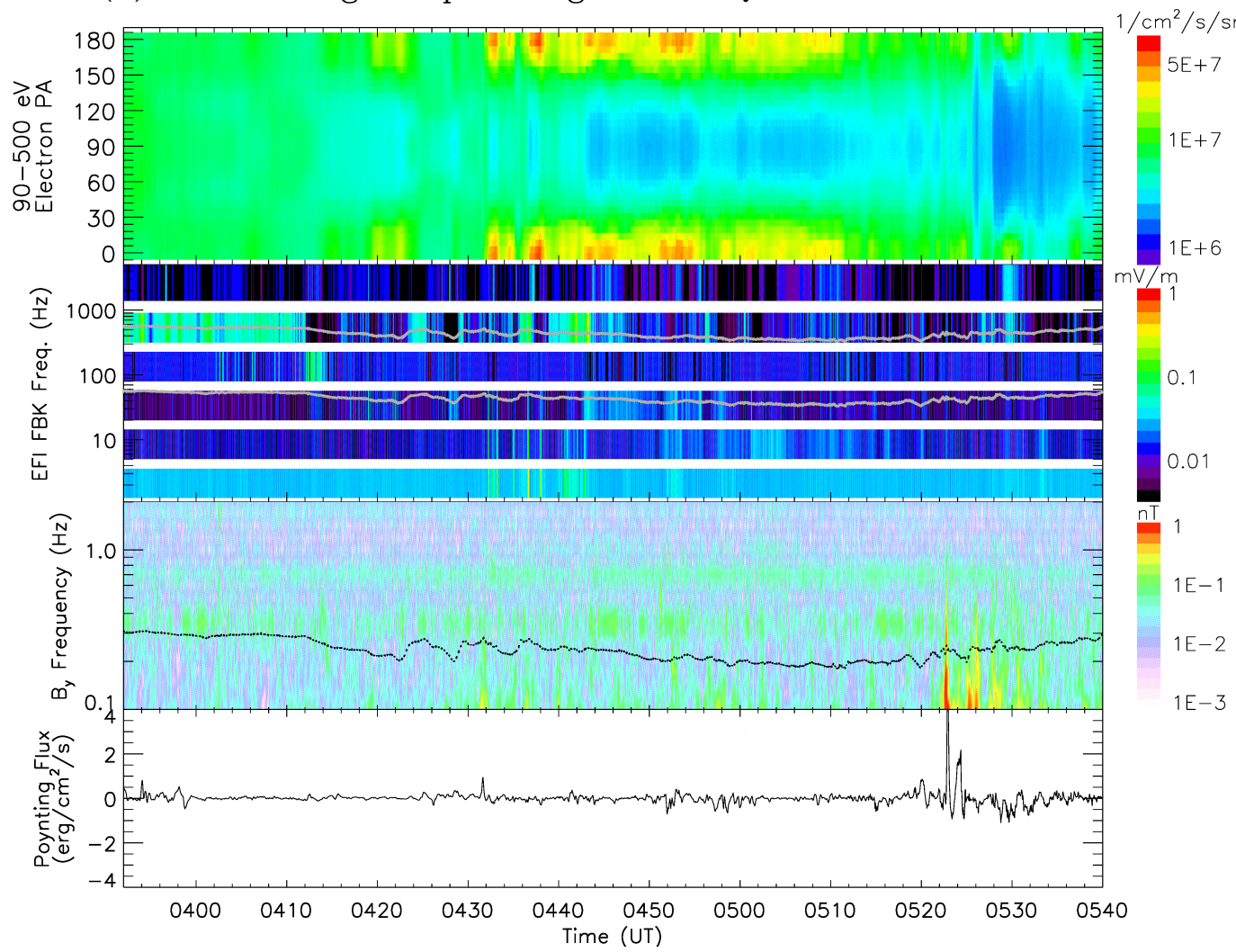

Fig. 5. (a) A few slices of the differential number flux spectra in parallel, antiparallel, oblique, and perpendicular directions, exemplifying the feature and evolution of the QPEB. The color code for the pitch-angle direction is labeled in the plot. (b) The top panel shows the pitchangle spectrogram of 90-500 eV electrons; the second panel shows the FBK data of EFI measurements; the third panel shows the wavelet scalogram of the $B_{\mathrm{y}}$ component from FGL dataset. The gray curves in the second and third panels denote $f_{\text {ce }}, 0.1 f_{\text {ce }}$, and $f_{\text {ci }}$, from high to low frequencies. The bottom panel shows the parallel Poynting flux mapped to the ionosphere.

activity, with no major substorm occurring during the interval of interest.

Figure 4 displays the in situ observations from TH-A. The top panel shows the magnetic field in GSM coordinate. $B_{\mathrm{Z}}$ is the dominant component, and the plasma $\beta$ (2nd panel) is well above 1 , indicating that the probe is within the CPS. The third panel shows the azimuthal and radial components of ion flows. The overall flow magnitude is moderate $\left(<80 \mathrm{~km} \mathrm{~s}^{-1}\right)$ with noticeable Pc5 ULF oscillations; the azimuthal flow component is stronger than the radial component, and is 
mainly directed duskward, as expected in a premidnight inner CPS. The bottom three panels present the directional electron energy flux spectrograms in perpendicular $(\alpha \sim 75$ $\left.105^{\circ}\right)$, parallel $\left(\alpha \sim 0-15^{\circ}\right)$, and antiparallel $\left(\alpha \sim 165-180^{\circ}\right)$ directions. The structures seen in the parallel and antiparallel spectrograms, during 04:32-05:11 UTC and between $\sim 100$ and $500 \mathrm{eV}$ energy range, constitute the QPEB. They are very pronounced in both the parallel and antiparallel directions, but hardly seen in the perpendicular direction. These QPEBs appear as "detached" structures, whose energy band is distinctly below that of the main thermal population of the ambient CPS electrons (in $\mathrm{keV}$ range), yet well above that of the ionospheric cold electrons. We note that before $\sim$ 04:24 UTC when the probe was at smaller radial distances, there are also discernible low-energy structures with bidirectional anisotropy. Those preceding quasi-parallel structures are composed of electrons with energies $<100 \mathrm{eV}$. As mentioned in the previous section, inferred from our FAST survey and existing literature, upgoing electron outflows with energies $<100 \mathrm{eV}$ at times randomly appear at latitudes equatorward of strong upgoing electron beam with energies $>100 \mathrm{eV}$, which is compatible with the magnetospheric observations shown in Fig. 4. Those low-energy structures at smaller radial distances, however, do not constitute the research object of this study, nor do they affect our main research efforts to demarcate of the tailward boundary of the QPEB region.

In Fig. 5a we present a few selected slices of the differential number flux spectra of electrons in parallel, antiparallel, oblique, and perpendicular directions. Before the appearance of the QPEB, the electron distribution is more or less isotropic at energies $\geq 100 \mathrm{eV}$. During the QPEB intervals, the parallel/antiparallel fluxes are dramatically enhanced and become much greater than the oblique and perpendicular fluxes in the energy range $\sim 100-500 \mathrm{eV}$. In particular, the flux spectra in the parallel/antiparallel directions distinctly show a "bump" structure maximizing at $\sim 180-300 \mathrm{eV}$. This bump spectrum hints at a parallel potential structure in the topside ionosphere as its source. The anisotropy is much less pronounced or virtually absent for energetic electrons $(>1 \mathrm{keV})$. The parallel and antiparallel fluxes appear to be essentially symmetric since the QPEBs originate from both hemispheres, and undergo bouncing motion when they are trapped in the magnetosphere. An equality between the parallel and antiparallel fluxes implies little or no net FAC in the equatorial CPS. We do occasionally see tiny yet perceptible differences between the parallel and antiparallel fluxes - e.g., the 04:46:30 UTC (also somehow in 05:04:59 UTC) frame in Fig. 5a, in which the parallel flux is slightly larger than the antiparallel flux, implying the sense of downward FAC (TH$A$ is located south of the neutral sheet). However, during the event interval, the difference between the measured parallel and antiparallel fluxes is found to be generally much less than $\sim 10 \%$ of the QPEB fluxes themselves, and cannot be used to reliably deduce the FAC considering the instrument resolu- tion. When the QPEB structure quenches after 05:11 UTC, the overall electron distribution tends to resume its nearly isotropic character. In the top panel of Fig. 5b we present the pitch-angle spectrogram integrated over the energy range 90-500 eV, which again clearly manifests the QPEB feature. The cone angles of the observed QPEBs during the interval 04:32-05:11 UTC are well within $\sim 20^{\circ}$ in both the parallel and antiparallel directions.

Many key features of our observed QPEBs are essentially consistent with those in the existing literature. For example, the energy range $(\sim 100-500 \mathrm{eV})$, the peak energy flux level $\left(\sim 10^{8} \mathrm{eV} \mathrm{cm}^{-2} \mathrm{~s}^{-1} \mathrm{sr}^{-1} \mathrm{eV}^{-1}\right)$, and the beam width $\left(<20^{\circ}\right)$ of our observed QPEBs are all compatible with the results from a statistical survey by Abel et al. (2002b) based upon CRRES measurements in the nightside inner magnetosphere. However, before we claim that those QPEBs are the magnetospheric trace of the upgoing electron beams from the ionosphere, we need to carefully examine other candidate mechanisms capable of producing electrons with parallel/antiparallel anisotropy. For example, Shiokawa et al. (2003) studied the bidirectional electron pitch angle anisotropy in the near-Earth plasma sheet, and concluded that the major source of bidirectional electrons in the CPS lies in the vicinity of the neutral sheet, including Fermi acceleration. We note however that, in our event, the observed QPEB exists as a "detached" structure whose energy band is distinctly below the CPS thermal population. We have checked the ion spectrograms (not shown) and found that similar QPEB structure does not exist for ions. The above observations would sufficiently exclude the possibility of adiabatic Fermi acceleration as the cause of the observed QPEBs. On the other hand, the fact that the parallel flux and antiparallel flux are essentially symmetric throughout the QPEB interval hints that the QPEB is unlikely to result from a local parallel electric field structure, such as a double layer (Ergun et al., 2009), close to the probe location in the CPS. Furthermore, the perpendicular scale of double layers in the CPS is expected to range from a few tens of $\mathrm{km}$ to a couple of hundred $\mathrm{km}$ (Ergun et al., 2009), while the QPEB structures are observed over extended time interval ( $30 \mathrm{~min}$ ), implying that their overall spatial dimension is much larger. Therefore, localized double layers in the CPS are unlikely to constitute a major mechanism of the observed QPEBs. Wave-particle interaction could be another candidate acceleration mechanism. For example, the kinetic Alfvén wave (KAW), the whistler-mode wave, and the electron cyclotron harmonic $(\mathrm{ECH})$ wave are all recognized as capable of accelerating ambient electrons at certain energy ranges, contingent upon the resonant conditions. The ECH wave is an electrostatic emission with frequencies above the electron gyrofrequency $f_{\text {ce }}$. The most common manifestation of the electromagnetic whistler-mode wave in the inner magnetosphere is the chorus wave within the frequency range $0.1-0.9 f_{\text {ce }}$. Readers are referred to Thorne et al. (2010) and references therein for detailed descriptions of the chorus and 
ECH waves as well as their resonant conditions with electrons. The parallel heating/acceleration of electrons by KAW can occur in two recognized ways: via Landau damping, and via a parallel electric field. To result in a parallel heating of ambient electrons via Landau damping, KAW must feature very large $k_{\perp}$ (Roux et al., 2011). Using the KAW dispersion relation in Roux et al. (2011) with observed local plasma parameters, we estimate that, to be able to interact with $\sim 100 \mathrm{eV}$ electrons via Landau resonance, $k_{\perp}$ must be on the order of $\sim 0.1 \mathrm{~km}^{-1}$. With the observed flow magnitudes of several tens of $\mathrm{km} \mathrm{s}^{-1}$, had such short-scale KAW existed, it would have been Doppler-shifted up to $\sim 1 \mathrm{~Hz}$ range. Based upon the above notions, in the second and third panels of Fig. $5 \mathrm{~b}$ we present the EFI FBK data in the frequency range $\sim 2 \mathrm{~Hz}-3 \mathrm{kHz}$, and the wavelet scalogram of the $B_{\mathrm{y}}$ component (other components of the $\mathrm{B}$ field are also examined) from the FGL dataset in the frequency range $0.1-2 \mathrm{~Hz}$. The two panels present an overview of electric/magnetic wave activities from below $f_{\text {ci }}$ to above $f_{\text {ce }}$. As one can see, during the QPEB interval, the overall wave activities are weak to moderate and sporadic only, and show little correlation with the QPEBs (except for $f \sim 10 \mathrm{~Hz}$, where a weak correlation is arguably present). On the other hand, the parallel $\mathrm{E}$ field contained in intense KAWs might be able to accelerate electrons up to $\sim 1 \mathrm{keV}$ (e.g., Wygant et al., 2002), but those intense KAWs and the resulting electron acceleration are favored to occur in the high-latitude magnetosphere rather than in the equatorial CPS where the plasma $\beta$ is high (see, e.g., discussions in Angelopoulos et al., 2002), and thus cannot be analyzed from local THEMIS data. Those short-scale KAWs at high-latitude magnetosphere are, however, supposed to be generated from the larger-scale Alfvén waves presumably originating near the Equator (Wygant et al., 2002; Angelopoulos et al., 2002). In this regard, we present the parallel Poynting flux $S_{/ /}=(\delta \boldsymbol{E} \times \delta \boldsymbol{H}) \cdot \hat{\boldsymbol{b}}$ in the bottom panel of Fig. 5. We have high-pass-filtered the TH-A electric and magnetic fields for periods $<10 \mathrm{~min}$ to obtain $\delta \boldsymbol{E}$ and $\delta \boldsymbol{H}$, and map $S_{/ /}$to the ionosphere $(B \sim 50000 \mathrm{nT})$. Again, the low-frequency Alfvénic Poynting fluxes show little correlation with the QPEB (the pronounced $B_{\mathrm{Z}}$ oscillations around $\sim$ 04:30 UTC is compressional rather than Alfvénic). The strongest $S_{/ /}$arises only after the QPEBs have disappeared. We have also checked the $\delta \boldsymbol{E}$ and $\delta \boldsymbol{B}$ data separately and found that neither of them have apparent correlation with the QPEB. An excitation of KAW by shear Alfvén waves of equatorial origin during the QPEB interval is thus not supported. Combining the above observations and considerations, we conclude that the wave-particle interaction appears not to be a promising mechanism for generating the observed QPEBs in this event. The upgoing electron beams of ionospheric origin stand out as the most likely source of the observed QPEBs.

Figure 6 presents the optical auroral observations from the FSMI MSP. The scanning meridian of FSMI MSP is $\sim 10^{\circ}$
MLON west of TH-A during the QPEB interval. The existence of an arc structure near the equatorward border of the auroral oval can be seen from the green-line $(557.7 \mathrm{~nm})$ auroras in the top panel of Fig. 6a. A brief episode of arc activation exists at $\sim 04: 33$ UTC in the $557.7 \mathrm{~nm}$ MSP data and fades at $\sim 04: 38 \mathrm{UTC}$, which is possibly associated with the QPEBs seen by TH-A during 04:32-04:38 UTC. A more intense and persistent arc structure reappears at $\sim 04: 45$ UTC, and gradually moves southward. Albeit with ULF modulations on its intensity, the arc structure persists until $\sim$ 06:00 UTC and possibly beyond.

The bottom panel of Fig. 6a shows the observations of the $H_{\beta}(486.1 \mathrm{~nm})$ line, which is excited by proton precipitation. A careful comparison between the latitudes of the peak band of the proton aurora and the arc structure reveals that the former consistently lies equatorward of the latter. In Fig. $6 \mathrm{~b}$ we show a few examples of the latitudinal profiles of green-line and proton auroral intensities. All frames consistently illustrate that the arc, depicted by a narrow peak of the green-line electron auroras, is located at the "poleward shoulder" of the proton auroras. Such a scenario is verified on an event-toevent basis whenever an equatorward-most arc and the proton aurora were concurrently observed (e.g., Samson et al., 1992; Donovan et al., 2012), and is also repeatedly confirmed by FAST measurements in that the inverted-V arc is always located slightly poleward of the most intense proton precipitation region (Dubyagin et al., 2003; Jiang et al., 2012).

More definite observations of the arc structure come from the MSI. To avoid dayglow contamination the FSIM MSI was scheduled to run after 04:38 UTC. Therefore, those earlier auroral activations seen in the MSP measurements, which are possibly related to the QPEB structures before 04:38 UTC, were not available on MSI. In Fig. 7 we present a few selected MSI images of $557.7 \mathrm{~nm}$ observations. The ionospheric footprint of TH-A, calculated from T02 model with realistic solar wind parameters, is overplotted on each auroral image for reference. Under the notion that the QPEBs originate from the ionosphere, we average the electron flux spectra within $5-15 \mathrm{~s}$ after each $557.7 \mathrm{~nm}$ image is taken in order to allow for a transit time for soft electrons from the ionosphere to reach the equatorial magnetosphere (note that $\sim 10.7 \mathrm{~s}$ is required for $100 \mathrm{eV}$ electron to travel along a $10 R_{\mathrm{E}}$ field line), and plot the resulting spectra to the right of each auroral image. Initially, the auroral morphology around the footprint of TH-A at $\sim 04: 39$ UTC (Fig. 7a) is characterized by some weak diffuse auroras without any clearly identifiable arcs. In situ observations reveal that both the flux and the pitch-angle anisotropy of 100-500 eV electrons are moderate, probably as a residual of previous QPEB activations. The first sign of an arc formation near the satellite footprint becomes barely discernible at $\sim 04: 41$ UTC (Fig. 7b), and the QPEB structure, characterized by a bump in the $\sim 100-500 \mathrm{eV}$ energy range of the parallel/antiparallel flux spectra, begins to develop. From then on, the arc gradually intensifies, and both the parallel/antiparallel flux and the 


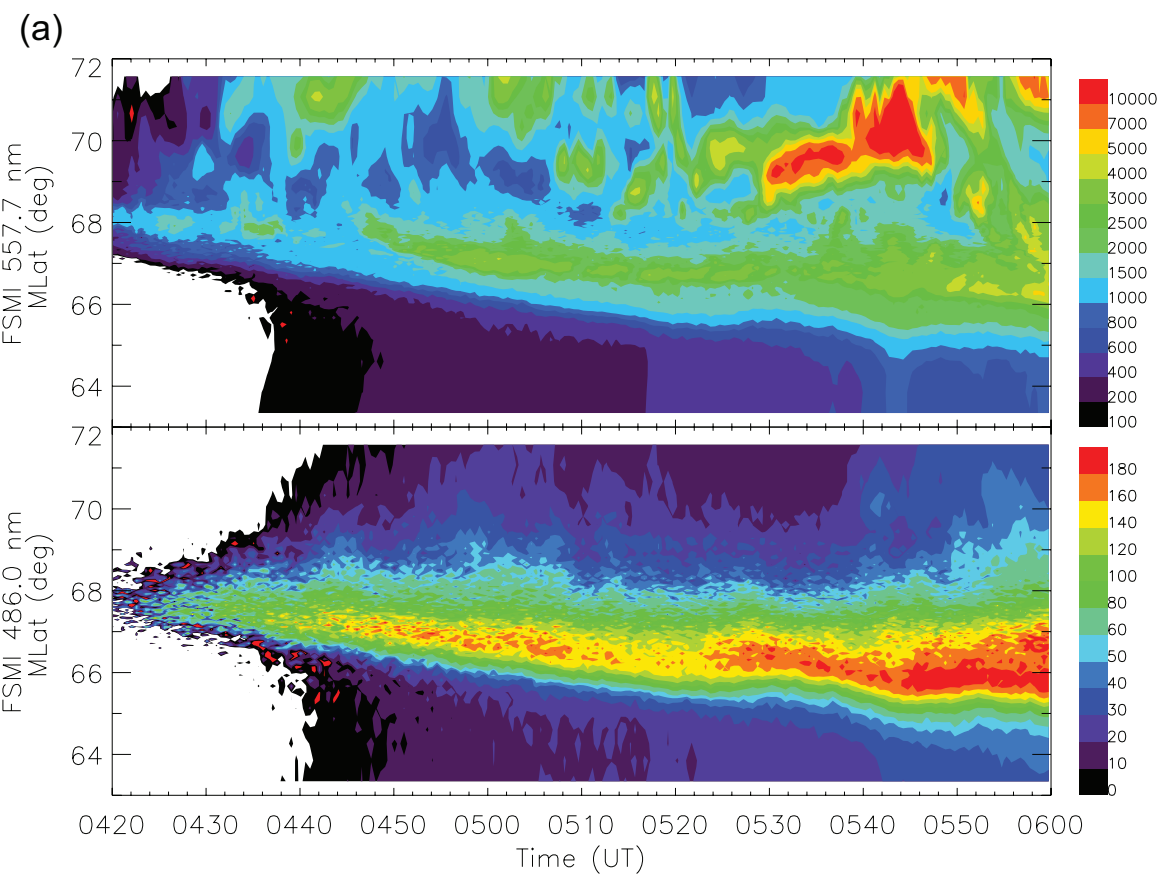

(b)
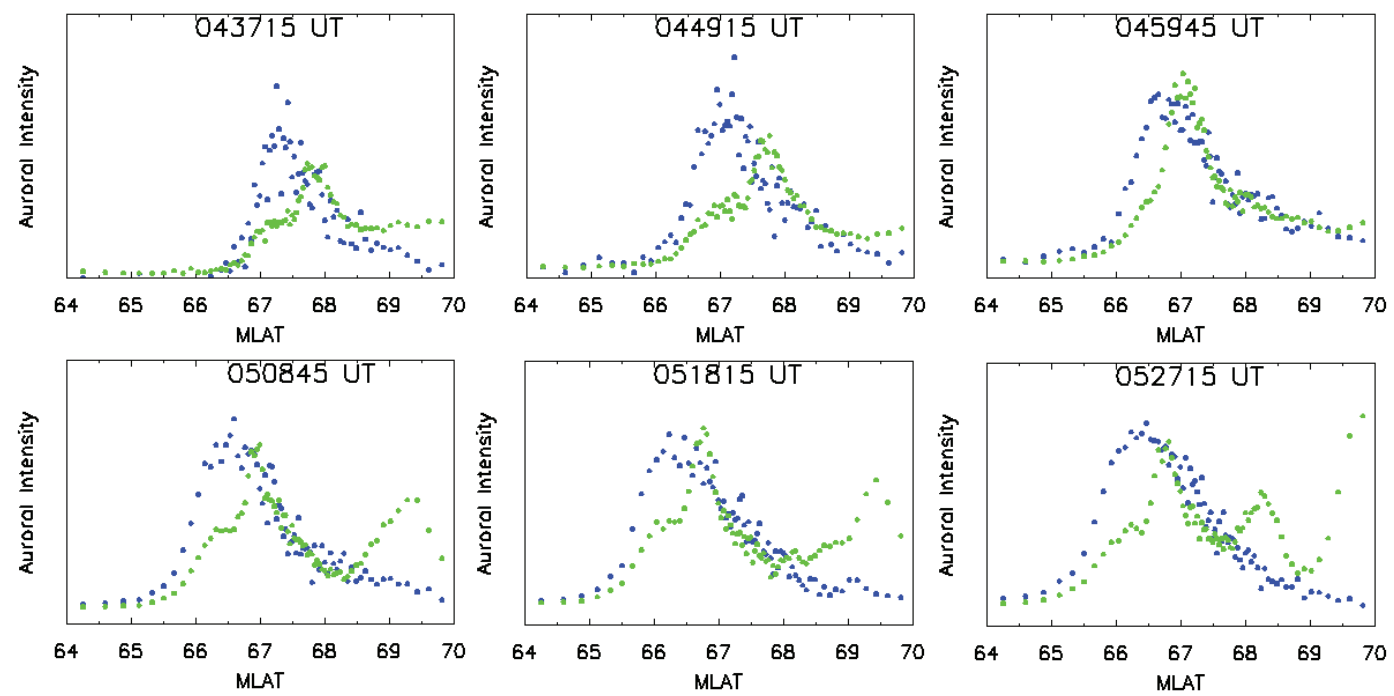

Fig. 6. (a) FSMI MSP observations of the green-line aurora (557.7 nm, top panel) and proton aurora (486.1 nm, bottom panel). (b) A few example slices showing the latitudinal distribution of $557.7 \mathrm{~nm}$ (green dots) and $486.1 \mathrm{~nm}$ (blue dots) auroral intensities. The intensities of two emission lines are scaled differently in the plot, such that only their latitudinal profiles are informative. Each dot represents the measurement from a latitudinal bin of the MSP.

anisotropy of $\sim 100-500 \mathrm{eV}$ electrons are enhanced. Note that at $\sim 04: 56$ UTC (Fig. 7e) the arc temporarily fades a bit in its eastern portion that is azimuthally close to TH-A. Coincidentally, a moderate fading is observed in the parallel/antiparallel flux, and the QPEB structure shifts toward lower energies. Note that a shift of the QPEB towards a lower energy range hints at a reduction of the downward parallel potential drop in the topside ionosphere which, for a given current-voltage relationship, implies a reduction of the downward FAC intensity. Such a brief dip of the QPEB feature at $\sim 04: 56$ UTC can also be seen from the top panel of Fig. 5b. Both the arc and the QPEB recover at $\sim 04: 58 \mathrm{UTC}$ (Fig. 7f) and continue their presences afterwards. To summarize, the observations unveil a good correspondence between the QPEB and the arc activation until 05:10 UTC, reinforcing the notion that the QPEBs carry the return current of the 

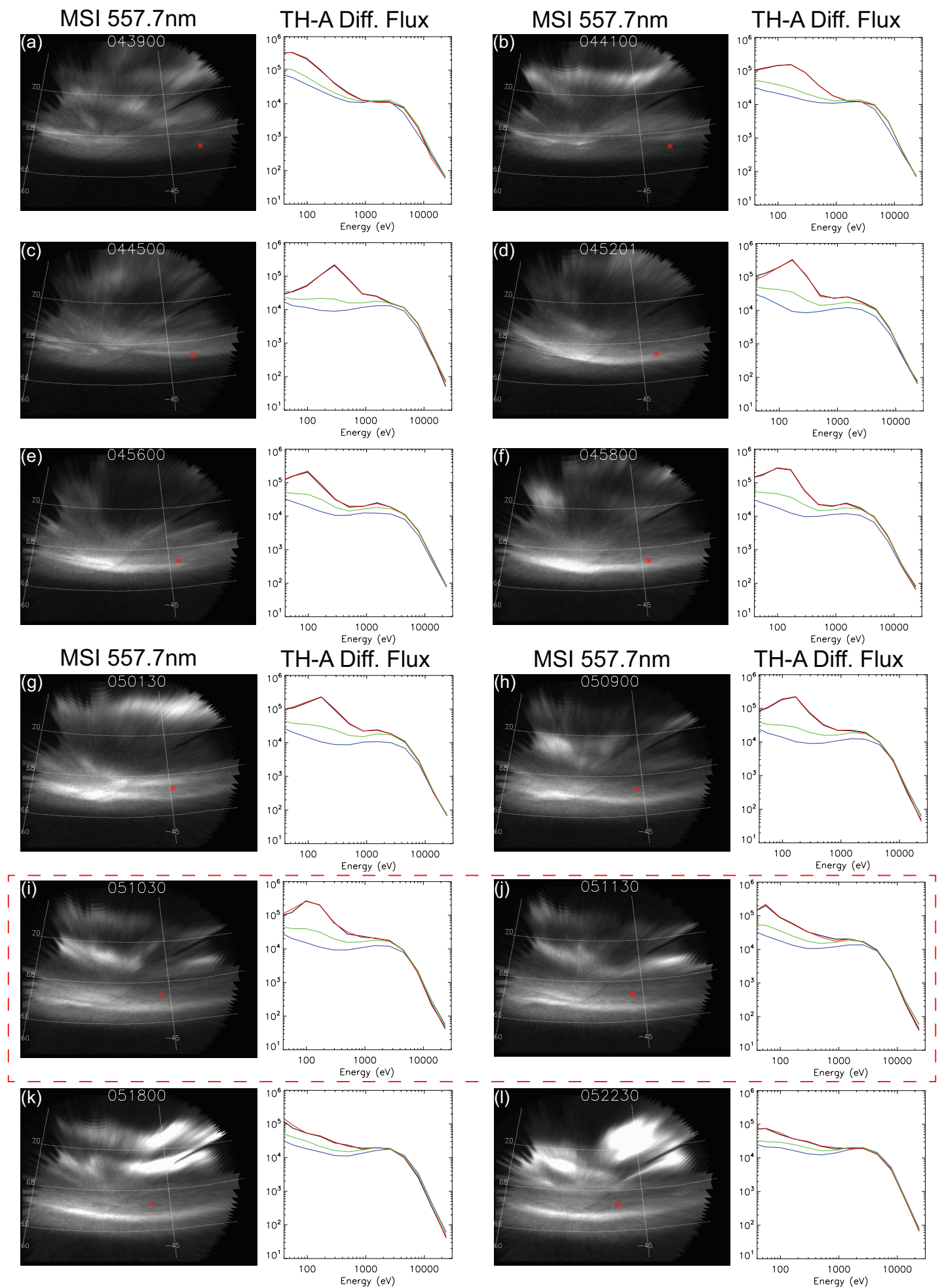

Fig. 7. A few selected images from FSMI MSI $557.7 \mathrm{~nm}$ observations. The images are converted into AACGM coordinates assuming $110 \mathrm{~km}$ emission height. The footprint of TH-A, calculated from T02 model, is plotted as a red asterisk in each image. To the right of each image is the correspondent electron number flux spectra observed by TH-A. The color code of the spectra plot is the same as in Fig. 5a. A red dashed box highlights the interval when the probe is traversing the boundary between QPEB and AR. 


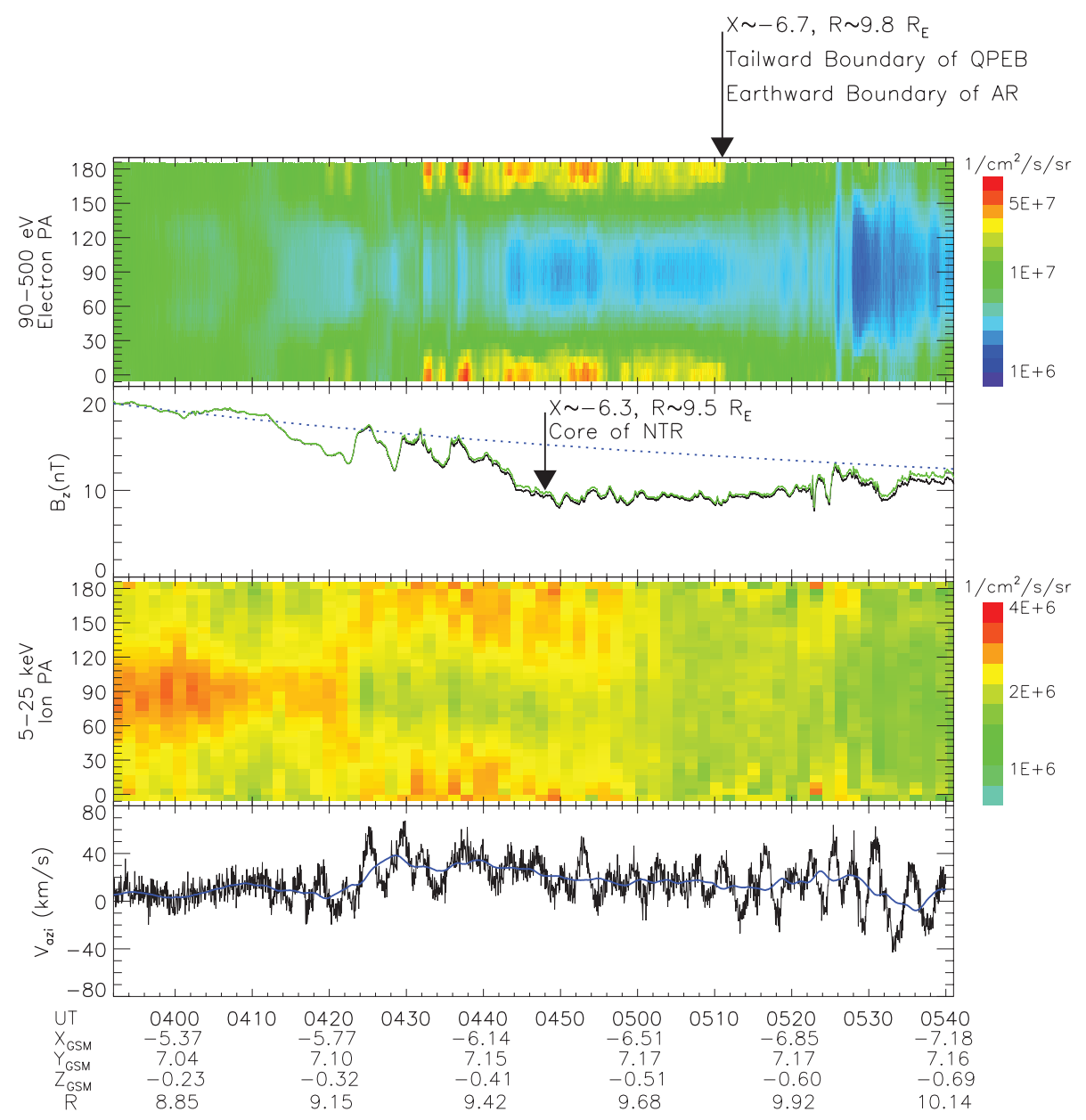

Fig. 8. Top panel shows the pitch-angle spectrogram of $90-500 \mathrm{eV}$ electrons; a tailward boundary of the QPEB region is marked (see text for details). The second panel shows the $B_{\mathrm{Z}}$ components (black curve) and the equatorial $B_{\mathrm{Z}}$ (green curve; see text for details) estimated from local observations; a dotted line indicates the $R^{-3}$ drop tendency of $B_{\mathrm{Z}}$ expected for a pure dipole field. The third panel shows the pitch-angle spectrogram of 5-25 keV ions from ESA measurements; the fourth panel shows the azimuthal (positive duskward) ion flows. A blue curve denotes the low-passed DC component of the flows.

arc FAC. The oblique and perpendicular fluxes show much less noticeable variations during the interval.

The correspondence between the QPEB and the arc are, however, disjointed after $\sim 05: 11$ UTC. Such a change is best seen upon a comparison between the 05:10:30 UTC and the 05:11:30 UTC frames (Fig. 7i-j): though there is no much variation of the arc itself, the QPEB feature in the TH-A flux spectra is heavily quenched in this $1 \mathrm{~min}$ interval. Afterwards, the arc continues its presence and, as a matter of fact, may even be slightly intensified (Fig. 7k-1), but the QPEB structures of our interest no longer exist.

The interpretation of the termination of the QPEBs at $\sim$ 05:11 UTC is critical for our study. Based on Fig. 7 we infer that such a termination is a spatial effect due to the radial motion of the satellite instead of a temporal effect related to auroral variations. This is corroborated by the observations of Fig. 4, which show that there are no substantial changes of the B fields or flows around $\sim 05: 11$ UTC, excluding a dramatic magnetospheric reconfiguration (or mapping) as the cause of the QPEB turn-off. Referring to the FAST observations and our proposed scenario in Fig. 2, we suggest that the probe starts to enter the AR at $\sim 05: 11$ UTC, due to an outward motion of the probe and/or (more likely) an inward migration of the AR. In other words, the epoch $\sim 05: 11$ UTC marks the tailward boundary of the QPEBs and correspondingly a possible earthward boundary of the AR.

We shall then examine the relative location of the QPEB region in the nightside magnetosphere. The top panel of Fig. 8 reproduces the pitch-angle spectrogram of $90-500 \mathrm{eV}$ electrons as previously shown in Fig. 5b, with the tailward boundary of the QPEB region labeled. The second panel shows the $B_{\mathrm{Z}}$ component on TH-A. During the event interval there is a mildly increasing trend of $\left|B_{\mathrm{x}}\right|$ (see Fig. 4), implying a probe motion away from the neutral sheet and/or a 
gradually stretching current sheet. Nevertheless, the $B_{\mathrm{x}}$ magnitude remains to be smaller than $B_{\mathrm{z}}$ during the interval of interest, and the plasma $\beta$ is high, such that the probe presumably stays well within the current sheet. Under such a geometry, the local $B_{\mathrm{Z}}$ would not differ substantially from its equatorial value. To support our argument we estimate the equatorial $B_{\mathrm{Z}}^{\text {eq }}$ using the Wolf et al. (2006) model (see their Eq. 9) and overplot it in Fig. 8. As expected, there are only small differences between the estimated $B_{\mathrm{Z}}^{\mathrm{eq}}$ and the local $B_{\mathrm{Z}}$ during the interval of interest. We conclude that a mild tendency of the probe to move away from the neutral sheet would not impose uncertainties on our following analyses of the variation trend of $B_{\mathrm{z}}$. As one can see, $B_{\mathrm{z}}$ first shows a long-standing decreasing trend with $R$ distance before $\sim$ 04:48 UTC, with a descending slope steeper than $R^{-3}$ (see dotted line for reference), and then becomes rather stable at $\sim 10 \mathrm{nT}$, nearly independent of radial distance. We have also checked the data prior to the event interval and confirmed that the deceasing trend of $B_{\mathrm{Z}}$ had been lasting ever since the probe entered the magnetosphere. The observation strongly suggests that the probe is undergoing a transition from a quasi-dipole field to a highly stretched current sheet. In accordance with Donovan et al. (2012), hereafter we shall abbreviate such a transition region as NTR (nightside transition region). We shall argue that the spatial/temporal ambiguity involved in the in situ measurement would not fundamentally affect our interpretation regarding the NTR. The event occurred during an extended interval of southward IMF $B_{\mathrm{Z}}$, such that the magnetic field presumably underwent a gradual stretching. We however note that, a gradual stretching of the tail magnetic field topology is naturally accompanied by an earthward migration of the NTR. In this regard, it remains valid to use the in situ data to infer a traverse over the NTR, under the notion that such a traverse comprises a combined consequence of the outward motion of the probe and the inward migration of the NTR itself. The NTR of course spans a finite radial extent, but an inward edge of the stretched current sheet region, or equivalently, the periphery of a quasi-dipole magnetosphere, can be estimated as where the most notable change of the $B_{\mathrm{Z}}$ gradients occurs - that is, $\sim 04: 48 \mathrm{UTC} ; X \sim-6.3 ; R \sim 9.5 R_{\mathrm{E}}$, as labeled in the plot. Compared to the above-inferred location of the earthward boundary of AR at $\sim 05: 11$ UTC; $X \sim-6.7$; $R \sim 9.8 R_{\mathrm{E}}$, even taking into account a slow migration of the NTR during the two measurement epochs, we are able to deduce the following key inference on the relative location of the AR with respect to the NTR: the AR itself is embedded in a highly stretched current sheet region with a relatively stable $B_{\mathrm{Z}}$, but its earthward edge is on the verge of a transition into a quasi-dipolar magnetosphere with much steepened $B_{\mathrm{Z}}$ gradients. The above inference of the AR location is fully consistent with a number of existing observations and theoretical proposals that an equatorward-most arc likely maps to the earthward portion of a stretched current sheet region, at the periphery of the quasi-dipolar magnetosphere (Lui and
Burrows, 1978; Galperin et al., 1992; Yahnin et al., 1997; Galperin and Bosqued, 1999; Sergeev et al., 2012).

The third panel of Fig. 8 shows the $5-25 \mathrm{keV}$ ion pitchangle spectrogram from ESA measurements. The ion pitch angle distribution is pancake shaped with larger perpendicular fluxes before $\sim$ 04:24 UTC, when the probes are deep inside a quasi-dipolar magnetosphere with strong $B_{\mathrm{Z}}$ gradients, and then become cigar shaped with larger parallel/antiparallel fluxes when the probe is approaching and traversing the NTR region. The change of ion distribution pattern can be readily interpreted by the adiabatic acceleration of thermal ions under different B-field topologies. In a quasi-dipolar magnetosphere, the variation of the equatorial B-field strength along the ion drift trajectory usually exceeds the change of the field-line length, leading to a stronger Betatron acceleration than Fermi acceleration and in turn a pancake-shaped distribution, while in the NTR there are substantial changes in the field-line length, such that the Fermi acceleration becomes important and leads to a cigar-shaped distribution. Subject to certain ambiguity of the pitch-angle scattering rate as discussed in previous section, the region of peak antiparallel (precipitation direction for TH-A) fluxes of $>5 \mathrm{keV}$ ions, taking place at $\sim 04: 25-04: 58 \mathrm{UTC}$, can be approximated as the region of peak proton precipitations, which manifests itself as the peak band of proton auroras in the ionosphere. Such peak region of proton precipitations is found as centered around the NTR, supporting the proposal of Donovan et al. (2012) that the peak band of proton auroras roughly marks the ionospheric projection of the NTR. On the other hand, the QPEB region is found to be partly colocated with and extend slightly tailward of the peak proton precipitation region. The relative geometry among the peak proton precipitation region, the QPEB, and the AR is thus consistent with the scenario shown in Fig. 2. The above observations and considerations also serve the purpose to resolve one ambiguity regarding the QPEB location with respect to the AR as mentioned in the previous section, namely whether the observed QPEB is earthward or tailward of the AR. For this event, inferred from the above analyses, were the observed QPEBs located tailward of an AR, the AR would be largely earthward of the peak proton precipitation region, which contradicts the optical observations shown in Fig. 6 that the arc is located distinctly poleward of the peak band of proton auroras.

The bottom panel of Fig. 8 shows the azimuthal component of the ion convective flows. We have removed the fieldaligned component in the data processing. To separate the DC component, namely the large-scale trend of the flows, from the ULF oscillations, we perform a 10 min SavitzkyGolay low-pass filtering of the flows, and show the outcome as the blue curve in the figure. Referring to the observations of QPEBs, $B_{\mathrm{Z}}$ and the ion fluxes shown in the above panels, one may see that the flows have a strong duskward DC component and large-amplitude ULF wave oscillations surrounding the NTR (or equivalently the peak proton precipitation 

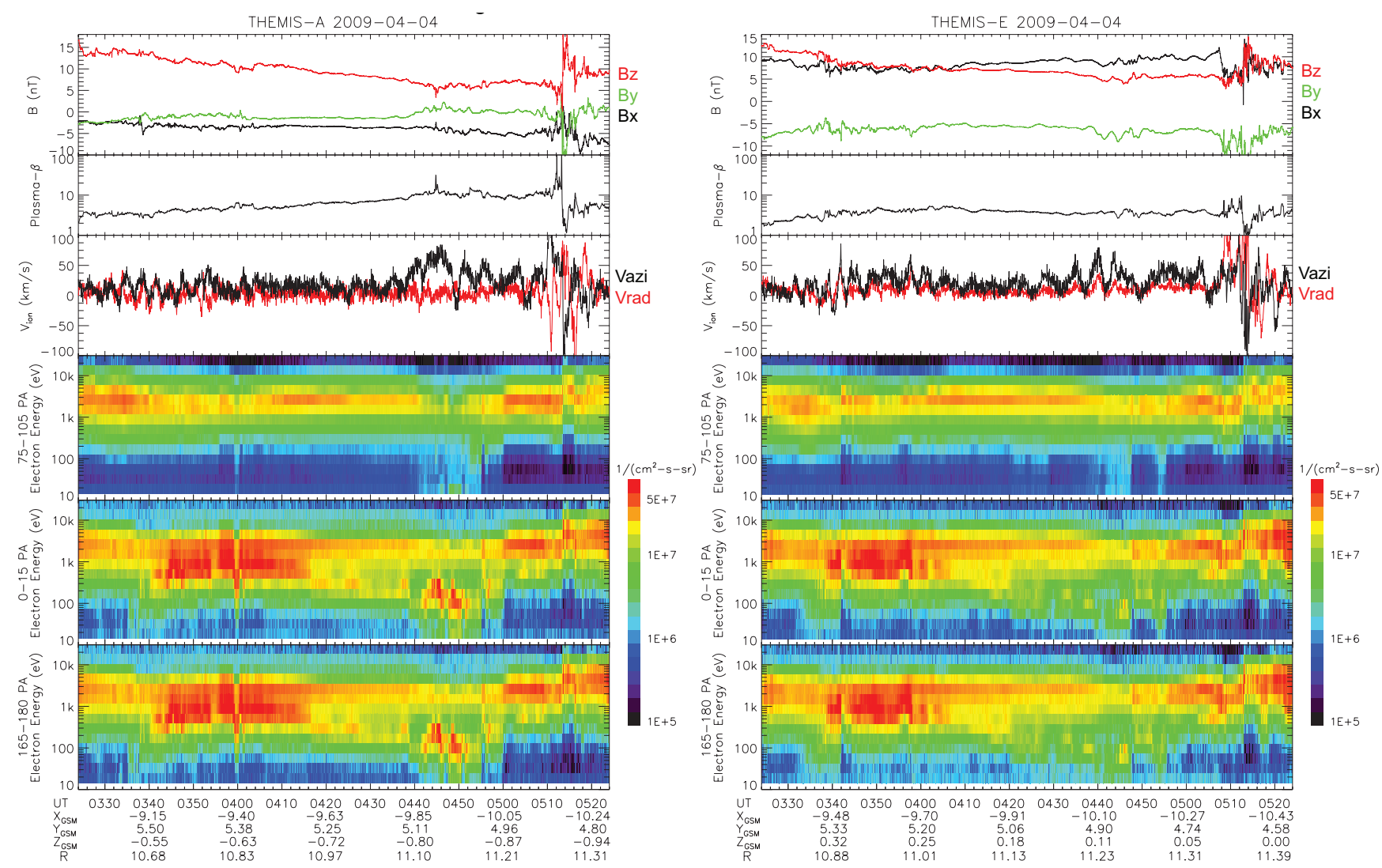

Fig. 9. (a) TH-A observations on 4 April 2009 in the same format as in Fig. 4. (b) TH-E observations during the same time interval and in the same plot format.

region), and in general gradually decrease in both DC flows and wave amplitudes toward the end of the QPEB region. When the probe enters the AR after $\sim 05: 11$ UTC, the azimuthal flows increase in ULF oscillation amplitudes again, possibly hinting at an association between shear Alfvén waves and the arc activation (e.g., Rönnmark and Hamrin, 2000), but with rather small DC components and even somehow dawnward deviations. The observations are compatible with the ionospheric observations that strong westward flows were found as embedded within intense proton precipitations equatorward of an auroral arc (Zou et al., 2009), and are also consistent with the results from a recent FAST survey on the E-field pattern surrounding the equatorward-most arc (Jiang et al., 2013) that a northward E-field (westward flow) peak is often found equatorward of the arc, while inside the arc the northward E field tends to reduce in magnitude, likely owing to the enhanced conductance therein (e.g., Marklund, 1984).

\subsection{April 2009 event}

Compared to Event 1, the satellite orbits of the 4 April 2009, 03:30-05:30 UTC event were more oblique - i.e., combining both radial and azimuthal motions. However, a notable advantage of the event lies in that the three inner probes $\mathrm{TH}$ -
A/E/D formed a close-spaced radial sequence. In particular, TH-A and TH-E were very close in radial and azimuthal distances, yet moderately separated in z-direction. The geometry helps us to deduce the spatial boundary of QPEB with better accuracy, and also allows for an estimation of several important local parameters such as the current intensity. The IMF $B_{\mathrm{z}}$ had been weakly negative $(>-1 \mathrm{nT})$ for hours before the event interval; one can thus expect that the ambient magnetosphere was much less stretched than in the previous event. The event interval of our main interest was during the growth phase of a small substorm with onset at $\sim$ 05:00 UTC.

Figure 9a shows the TH-A observations in the same format as Fig. 4. $B_{\mathrm{Z}}$ is the dominant component, while $B_{\mathrm{x}}$ remains small during the event interval, and the plasma $\beta$ is well above 1, indicating the probe is close to the neutral sheet. The flow features are essentially the same as in Event 1, namely containing moderate $\left(<100 \mathrm{~km} \mathrm{~s}^{-1}\right)$ duskward flows and much weaker radial flows before the substorm onset. The electron spectrograms reveal two prominent periods of QPEB intensifications. The first is during 03:40 04:16 UTC. For this intensification interval, the core energy of the QPEBs maximizes around $\sim 1 \mathrm{keV}$, close to the ambient CPS thermal electron population. Afterwards, QPEB 
structures are still visible but decrease in both flux intensity and energy range. After $\sim 04: 40$ UTC, the QPEBs intensify again, mainly in the energy range $100-500 \mathrm{eV}$, similar to that in Event 1. Figure 9b gives the TH-E observations. TH-E is in the northern CPS and slightly tailward of TH-A, and is more off the neutral sheet as inferred from the larger $B_{\mathrm{x}}$ magnitude and smaller plasma $\beta$ as compared to TH-A. The first interval of QPEB intensifications identified on TH-A after 03:40 UTC is also well seen on TH-E, with nearly identical energy range and flux level. With careful comparison one may find that the QPEBs appear slightly earlier on TH-E ( $\sim$ 03:38 UTC) than on TH-A ( $\sim 03: 42$ UTC), which is understandable since TH-E is slightly tailward of (and farther off the neutral sheet than) TH-A and thus enters the QPEB region earlier. However, the second interval QPEB intensification, shown on TH-A during 04:40-05:00 UTC, is only marginally seen on TH-E with much reduced flux level and persistency. This discrepancy will be analyzed in more detail later in this section.

The optical observations of the event are less ideal than those in Event 1. Firstly, the proton aurora was contaminated by moonlight during the interval, prohibiting us from using the proton precipitation region as a meaningful context for the arc as we did in Event 1. Secondly, in this event, the auroral arc is located near the edge of, or even outside, the field of view (FoV) of both MSP and MSI. The geometry is particularly problematic for the $557.7 \mathrm{~nm}$ auroras whose emission height is lower and thus the FoV is more limited. In this regard, we choose to use the red-line $(630 \mathrm{~nm})$ aurora whose emission height is higher and thus, for a given elevation angle, has larger latitudinal coverage. Since the $630 \mathrm{~nm}$ aurora features a broad range of emission height $(\sim 170$ $300 \mathrm{~km}$ ), for low-elevation-angle measurements the latitudinal resolution would be smeared out. Thus, we cannot precisely determine the latitude of the arc from the $630 \mathrm{~nm}$ observations. Figure 10a shows the keogram of $630 \mathrm{~nm}$ auroras from RANK MSI and GILL MSP, which clearly reveals the long-standing existence of an auroral arc. Though the arc is at exceptionally high latitude $\left(\sim 70^{\circ}\right.$ MLAT assuming a constant $230 \mathrm{~km}$ emission height), it still represents the equatorward-most arc near the equatorward border of auroral oval. The arc has two distinguishable activation periods: one during $\sim 03: 35-04: 15$ UTC, and the other during $\sim 04: 38$ 04:56 UTC. The two arc activation intervals roughly coincide with the two QPEB intensification intervals seen on TH-A/E, suggesting a connection between the arc activation and the QPEB intensification. Shortly after the second arc activation interval, the arc breaks up with strong brightening and poleward expansion, characterizing a small substorm onset. The expansion of the substorm auroras toward both south and north becomes prominent at $\sim$ 05:06 UTC. The in situ magnetic responses to the substorm onset are shown in Fig. 10b. Large-amplitude magnetic disturbances as signatures of the substorm are first seen on the outermost probe TH-D at $\sim$ 05:06 UTC, then 1-2 min later on TH-E,

\section{(a) $630 \mathrm{~nm}$ Keogram from RANK and GILL}
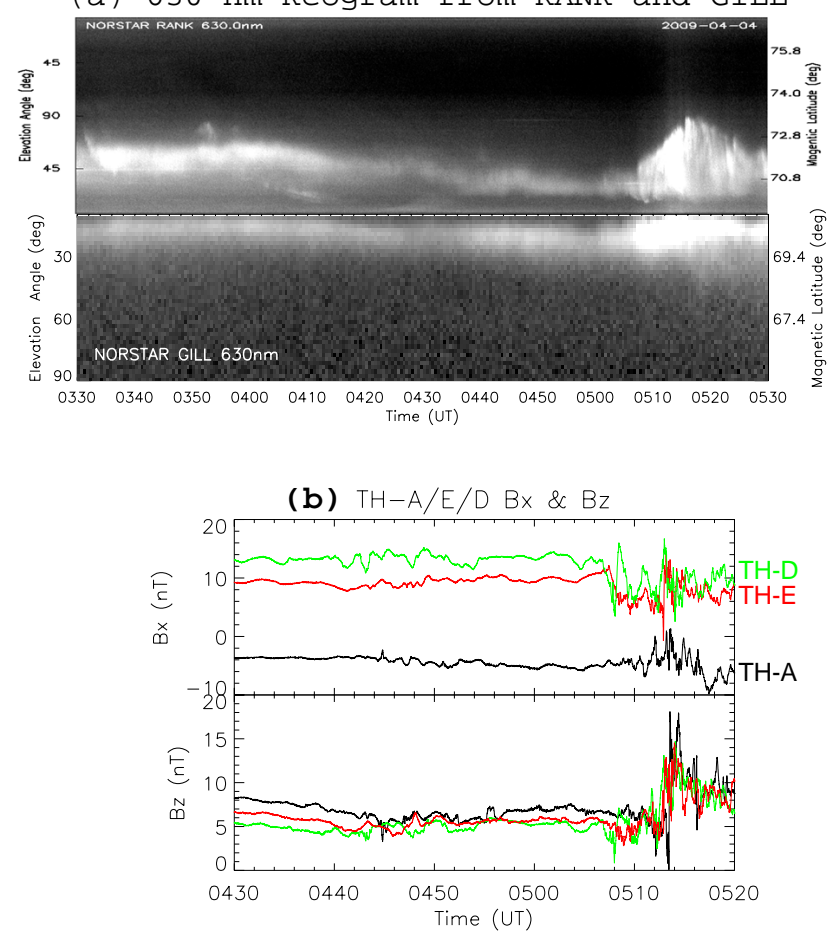

Fig. 10. (a) $630 \mathrm{~nm}$ auroral keograms from RANK MSI and from GILL MSP. Note that the arcs shown on two keograms are actually the same arc. We label the estimated MLATs as rightside tick by assuming a constant $230 \mathrm{~km}$ emission height, with the caveat that such estimation is subject to large uncertainty for low-elevationangle measurement. (b) The in situ $B_{\mathrm{X}}$ and $B_{\mathrm{Z}}$ components on THA (black), TH-E (red), and TH-D (green); an inward propagation sequence of substorm disturbance is inferred.

and lastly on the innermost probe TH-A after 05:10 UTC. Such a propagation sequence points to the scenario that the activation center of the substorm onset is initially tailward of TH-A/E, thereby excluding the possibility that the observed QPEB region is situated tailward of the onset arc.

In the following analyses, we limit our interest to the second interval of QPEB and arc activation ( 04:3804:58 UTC) for two reasons: (a) during the first arc activation interval the THEMIS probes are located east of the FoV of GILL MSI, while during the second activation interval the probes map well within the FoV of the imager such that a conjugate geometry is better achieved. (b) As we shall illustrate in more detail later, during the second arc activation the QPEB appearances on TH-A and TH-E are quite different, a feature allowing us to practically estimate the boundary of QPEB region. Furthermore, during the second arc activation interval, the QPEBs exist as "detached" structures whose energy bands are distinctly below the CPS main thermal population. The parallel flux and antiparallel flux associated with the QPEBs are essentially symmetric. The above features, as discussed in the previous event, hint that 
MSI 630.0nm

(a) 041800 UT

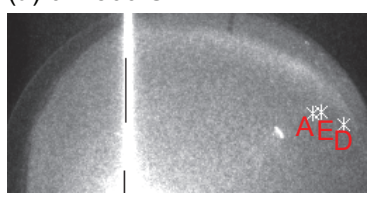

(c) 043801 UT

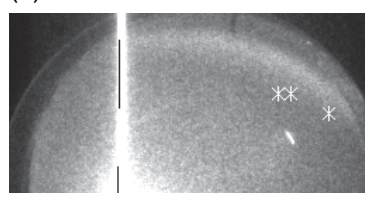

(e) 045031 UT

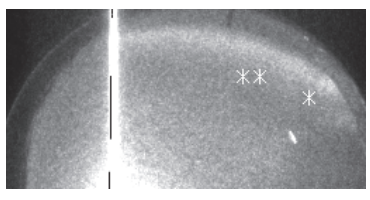

TH-A/E Diff. Flux
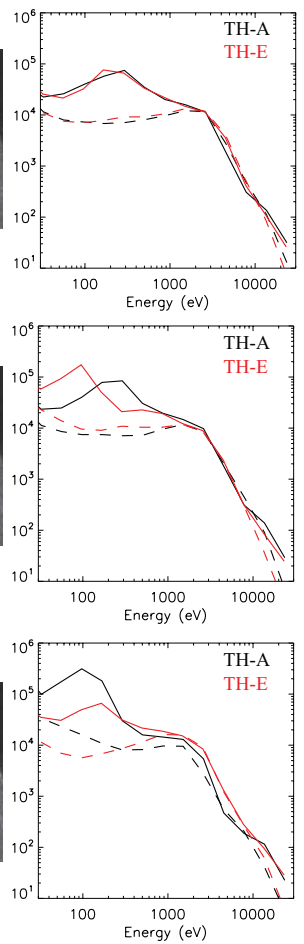

MSI 630.0nm

(b) 043200 UT

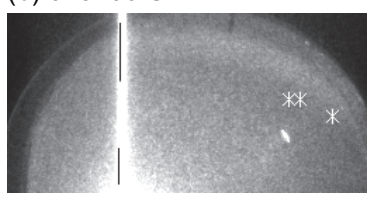

(d) 044500 UT
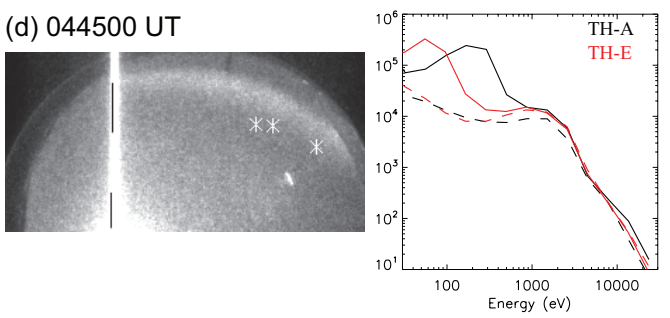

(f) 045630 UT
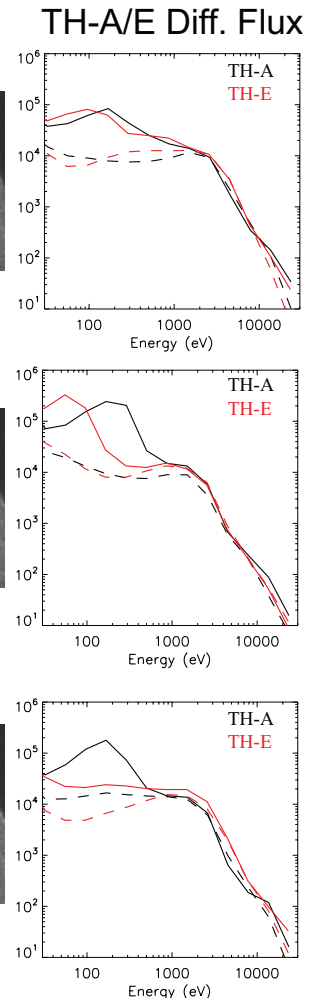

Fig. 11. A few selected images from GILL MSI $630.0 \mathrm{~nm}$ observations. In each image the up-direction denotes the geographical north and the right-direction denotes the geographical east. The footprints of TH-A/D/E, calculated from T02 model by assuming a $230 \mathrm{~km}$ mapping height, are plotted as asterisk for reference. To the right of each image is the corresponding electron number flux spectra, including the TH-A parallel spectrum (black solid curve), TH-A perpendicular spectrum (black dashed curve), TH-E antiparallel spectrum (red solid curve), TH-A perpendicular spectrum (red dashed curve).

the upward-accelerated electrons from the ionosphere are the most likely origin of the observed QPEBs. To examine the possibility of wave-particle interaction as a potential cause of the observed QPEBs, we have also checked the SCM and FBK wave data (not shown) in much the similar way as we did in previous event, and confirm that there is an absence of noticeable electromagnetic wave activity in the frequency range from $\sim f_{\mathrm{ci}}$ to $f_{\mathrm{ce}}$ that can be correlated with the observed QPEBs. However, during this event interval, the EFI boom was experiencing sphere shadowing, such that the EFI FBK data are contaminated, preventing us from checking the existence of electrostatic waves such as the ECH emission.

Figure 11 presents a few selected images of the MSI observations of $630 \mathrm{~nm}$ auroras and the corresponding differential flux spectra observed by TH-A and E. We again average the electron flux spectra within $5-15 \mathrm{~s}$ after the auroral image time to allow for a transit time of soft electrons from the ionosphere to reach the equatorial magnetosphere. Note that the format of Fig. 11 is somewhat different from that in Fig. 7: (a) in Fig. 11 we do not convert the arc into a geomagnetic coordinate due to the above-mentioned geometric difficulty, though we still overplot the footprint of the THEMIS probes for reference, assuming a $230 \mathrm{~km}$ map- ping height and projecting them on the camera surface. (b) In Fig. 11 we present the differential flux spectra on both TH$\mathrm{A}$ and TH-E. The probes gradually enter into the MSI FoV after $\sim 04: 14$ UTC. As an aftermath of the fist arc activation interval, at $\sim$ 04:18 UTC (Fig. 11a) there are still discernible bump structures at $\sim 100-500 \mathrm{eV}$ energies, characteristic of the QPEB feature, in the TH-A parallel and TH-E antiparallel flux spectra. By that time, the QPEB structures are fairly alike on TH-A and TH-E in terms of both flux level and energy range. There is a decreasing trend of the arc intensity afterwards (see also Fig. 10). By 04:32 UTC (Fig. 11b) the arc becomes relatively faint, and the bump structures of the in situ spectra become shallower and shift to lower energies, particularly on TH-E - we recall the notion that a shift of the QPEB toward lower energies and/or weaker fluxes would imply a reduction of the downward FAC intensity. The arc starts to reintensify at $\sim 04: 38 \mathrm{UTC}$ (Fig. 11c) and reaches a maximum at $\sim 04: 45$ UTC (Fig. 11d), and the QPEB feature on TH-A enhances accordingly. However, a dramatic difference exists between the QPEB structures on TH-A and TH-E: the QPEBs on TH-E are observed at lower energies (Fig. 11c, d), or with much weaker fluxes (Fig. 11e), than those on THA. The overall arc intensity starts to decrease mildly after 


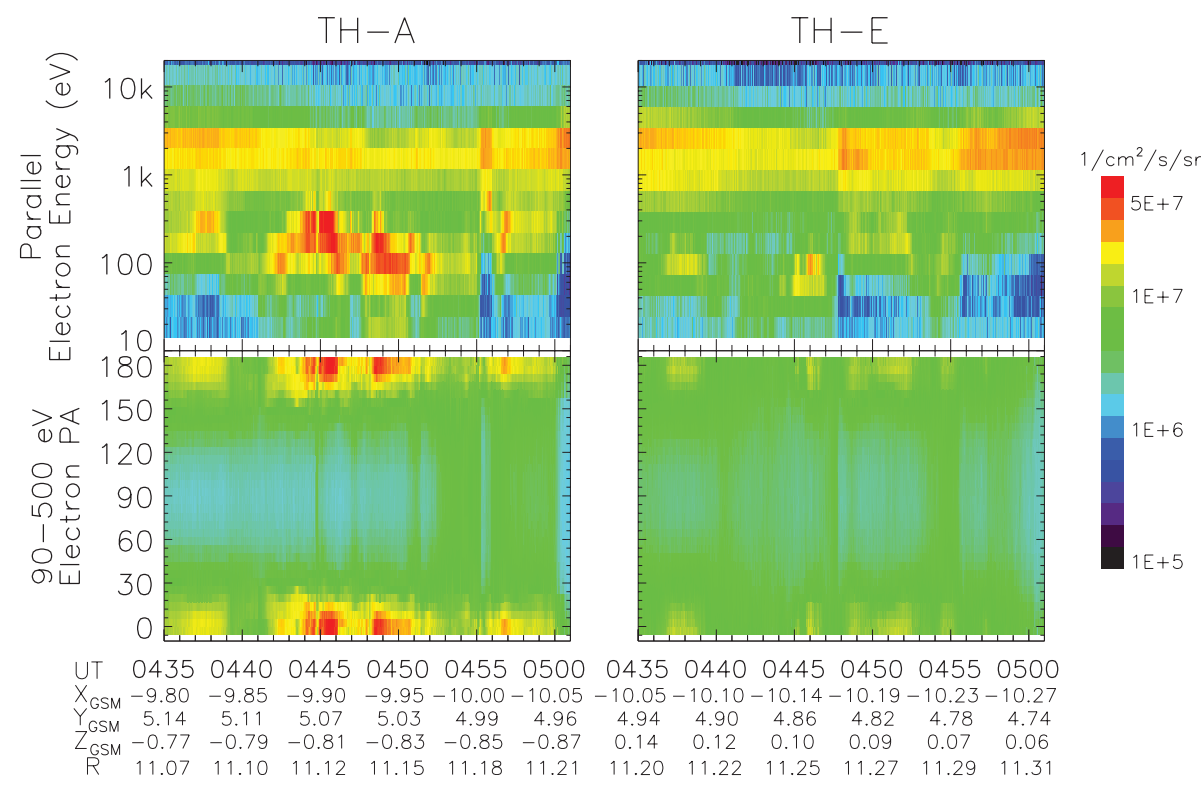

Fig. 12. Top panels show the electron energy flux spectrogram in parallel direction for TH-A (left side) and TH-E (right side) during the second arc activation interval. The bottom panels show the pitch-angel spectrogram of 90-500 eV electrons for TH-A and TH-E.

$\sim$ 04:56 UTC but the arc remains fairly visible. The QPEB feature is still pronounced in the TH-A parallel flux spectra by then, but entirely disappears on TH-E (Fig. 11f). As mentioned above, TH-A and TH-E are close in radial and azimuthal distances but moderately separated in the z-direction, such that TH-E is presumably mapped to higher latitudes in terms of ionospheric footprint (and equivalently farther radial distances in terms of equatorial footprint) than TH-A. The discrepancy of the QPEBs observed by TH-A and TH-E points to the scenario that, during the second arc activation interval, both TH-A and TH-E are situated near the outer edge of the QPEB region by then, where a sharp radial gradient of the QPEBs exists between TH-E and TH-A.

To further illustrate the above scenario, we present in Fig. 12 the differential energy flux spectrograms in the parallel direction as well as the pitch-angle spectrograms of $\sim 90-500 \mathrm{eV}$ electrons on TH-A and TH-E during the auroral activation interval 04:35-05:01 UTC. The QPEBs are clearly seen by the inner-most probe TH-A as distinctly detached structures $(\sim 100-500 \mathrm{eV})$ from the main CPS thermal population in the energy spectrogram, with collimated pitch-angle distribution. Those "detached" QPEB structures on TH-E are, however, much less pronounced: they reduce significantly in both intensity and persistency, and entirely vanish after $\sim 04: 55$ UTC. We have also checked the TH-D electron spectrogram (not shown) and confirmed that a detached QPEB structure of hundreds of eV energy is not identified by the outer-most probe TH-D. Based upon the above observations, we suggest that, during this arc activation interval, both TH-A and TH-E are located near an interface between the QPEB and the AR. TH-A essentially remains in the QPEB region earthward of the AR, while TH-E straddles, and eventually travels beyond, the outer boundary of the QPEB. In practice, we shall mark the epoch $\sim 04: 55$ UTC as the "boundary crossing" between the QPEB and AR by TH-E, when the arc is still active and the QPEB features are sustained on TH-A afterwards, but completely vanish on TH-E. Since TH-E is relatively off the equatorial plane, we trace it to the magnetic equator using T02 model, and estimate the boundary between QPEB and AR as $X \sim-10.3$; $R \sim 11.4 R_{\mathrm{E}}$ at $\sim 04: 55 \mathrm{UTC}$. The inferred radial location of the $\mathrm{AR}$ is somewhat larger than average (e.g., $\sim 7.5-11 R_{\mathrm{E}}$ as concluded in Sergeev et al., 2012), but is not unreasonable in this particular event, considering the fact that the arc itself is observed at exceptionally high latitude.

Based upon the above multiprobe analyses of QPEB features, Fig. 13a gives a schematic diagram showing the geometry of the probes with respect to the QPEB region and the AR. The multiprobe geometry in this event also offers us an ideal opportunity to examine the arc-related current system in the equatorial magnetosphere. To close the upward FAC tied to the arc and the accompanying downward FAC carried by the QPEB, closure currents must exist in the magnetosphere. The closure currents of the arc-related FACs would be presumably radially inward, opposite to the ambient $\mathrm{E}$ field, which tends to be radially outward in the duskside inner CPS. The geometry thus constitutes a dynamo $(\boldsymbol{J} \times \boldsymbol{E}<0)$ geometry of the arc-related FAC system. Readers are referred to Rothwell et al. (1991), Rönnmark (1999), and Haerendel (2007) to see the advocated geometry of the auroral current circuit and the underlying rationale. Such current geometry is also sketched in Fig. 13a. This arc-related current 


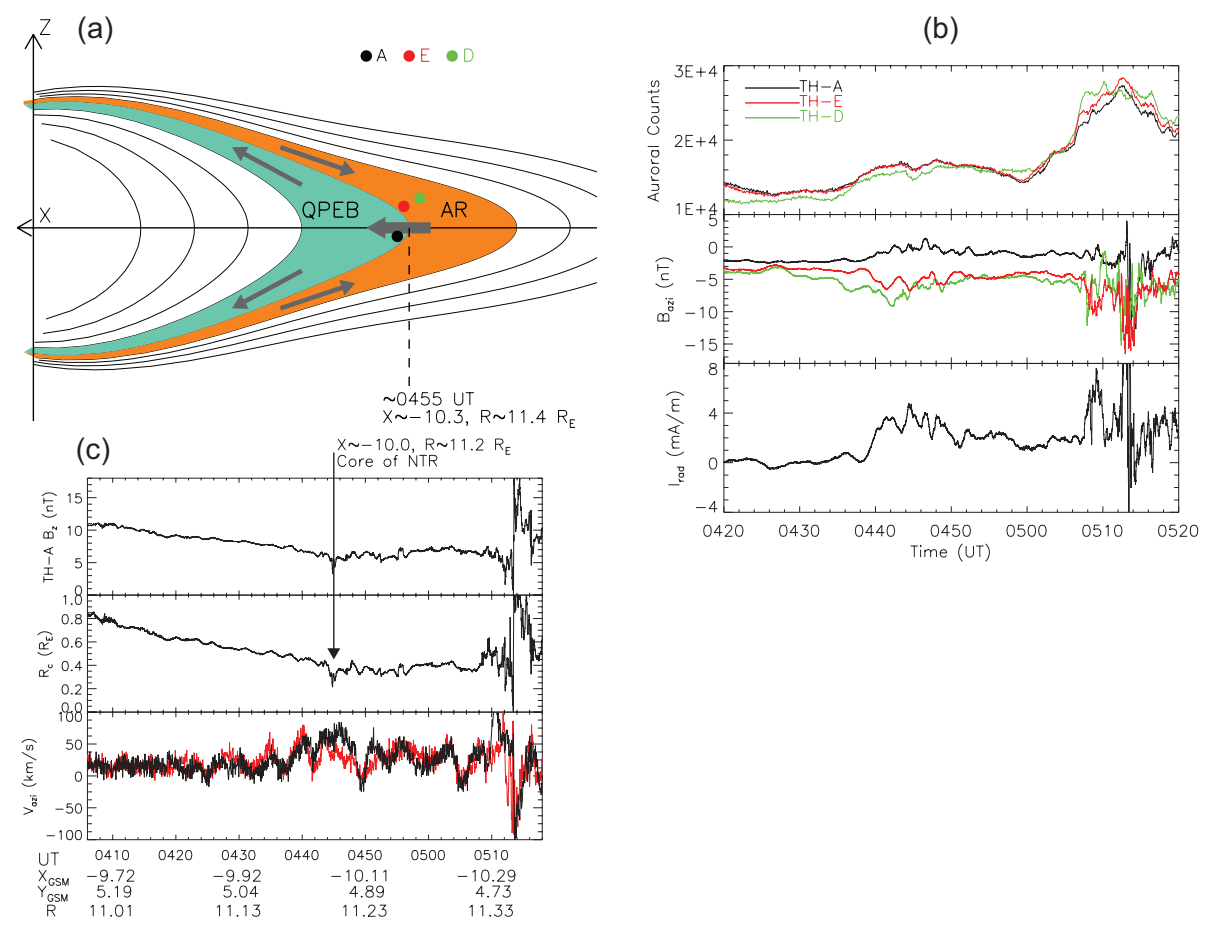

Fig. 13. (a) Schematic diagram illustrating the geometry of probes (dots with color code as labeled) with respect to QPEB and AR regions. The solid arrows mark the direction and geometry of the arc-related currents, including the FACs and their radial closure current. (b) Top panel shows the arc intensity sampled along the magnetic meridian $\left( \pm 2^{\circ} \mathrm{MLON}\right)$ of the footprint of each probe. The second panel shows the $B_{\text {azi }}$ component on each probe. The bottom panel shows the estimated radial current intensity between TH-A and TH-E. (c) Top panel reproduces the $B_{\mathrm{Z}}$ from TH-A observations. The second panel shows the radii of curvature of magnetic field $\left(R_{\mathrm{c}}\right)$ estimated from the measurements on TH-A and TH-E (see text for details). The "core of NTR" is estimated according to the abrupt change of the trend of $B_{\mathrm{Z}}$ and $R_{\mathrm{C}}$. The bottom panel shows the azimuthal ion flows on TH-A (black) and TH-E (red).

system would produce measurable signatures in the in situ B fields. Such magnetic effects are expected to be most significant in the transition region from QPEB to AR; they would be mainly azimuthal and reverse in polarity in northern and southern CPS, and are supposed to be zero right at the equatorial plane under symmetric condition. Such magnetic effect is hinted in Event 1: there the $B_{\mathrm{y}}$ component (see Fig. 4) shows a duskward deviation (note that TH-A is in southern CPS) when the probe is on its way leaving the QPEB region, but it is difficult to convincingly analyze the magnetospheric currents based upon single-probe measurements. Such a task can, however, be performed in Event 2 with a more favorable multiprobe geometry.

To help distinguish the temporal, azimuthal, and radial variations, we present in the top panel of Fig. 13b the arc intensity sampled along the magnetic meridian $\left( \pm 2^{\circ}\right.$ MLON) of the ionospheric footprint of each probe. We focus our interest on the prebreakup activation interval $\sim 04: 36-$ 05:00 UTC. The longitudinal distance between the footprints of TH-A and TH-E is smaller than the sampling range $\pm 2^{\circ}$ MLON, such that there is no discernible difference in the sampled arc intensities for these two probes. TH-D, however, is distinctly east of TH-A/E. As one can see, the start of the arc activation occurs slightly earlier on TH-D meridian than on TH-A/E meridian, indicating an azimuthal difference. On a detailed look into the MSI images in a $6 \mathrm{~s}$ cadence we confirm that the arc activation indeed initiates in the eastern portion of the arc, and propagates westward subsequently. In conjunction with the arc activation, perturbations of the azimuthal B fields ( $\left.B_{\text {azi }}\right)$ arise on all probes as shown in the second panel. Some salient features of the observed $B_{\text {azi }}$ variations are analyzed as follows:

1. The overall polarity of the $B_{\text {azi }}$ deviation is negative (dawnward) on TH-D and TH-E, while small yet positive (duskward) on TH-A. These patterns are fully consistent with the geometry shown in Fig. 13a: TH-E and TH-D are in the northern CPS, such that the magnetic effect led by the earthward-directed perpendicular current is dawnward. TH-A, on the other hand, is in the southern CPS but close to the neutral sheet; therefore a small duskward deviation of $B_{\text {azi }}$ is expected.

2. Since TH-A and TH-E are very close in radial and azimuthal distances but moderately separated in $z$ distance, the geometry enables us to estimate the total radial current intensity between TH-A and TH-E 
according to $I_{\mathrm{rad}} \approx\left(B_{\mathrm{azi}}^{\mathrm{TH}-\mathrm{A}}-B_{\mathrm{azi}}^{\mathrm{TH}-\mathrm{E}}\right) / \mu_{0}$. Albeit with noticeable ULF oscillations, the inferred $I_{\text {rad }}$ intensity shows apparent correlation with the arc brightness sampled along the meridian of TH-A/E during the arc activation interval 04:38-04:58 UTC, strongly suggesting a close relationship between the arc activation and the radial current intensification as the closure of the arc-related FACs. More quantitatively, if we further assume that such radial currents fully diverge into the FACs and use an azimuthal mapping factor of $\sim 24$ between the ionosphere and the equatorial CPS as inferred from T02 model, a $\sim 4 \mathrm{mAm}^{-1}$ peak radial current intensity would convert into a latitudinally integrated FAC of $\sim 0.1 \mathrm{Am}^{-1}$ in the ionosphere, which matches the order of magnitude of a typical arc FAC intensity. Under the notion that the radial closure current is mainly operative in a transition region from the QPEB to AR, the above observations also imply that during the arc activation interval TH-A/E are within or close to such a transition region, which is consistent with the aboveinferred geometry of TH-A/E with respect to the earthward boundary of AR based upon their QPEB features.

3. In terms of the timing among probes, the $B_{\mathrm{azi}}$ deviation commences earlier on TH-D than on TH-A and TH-E. This time difference, upon a comparison with the concurrent auroral observations shown in the top panel, is mainly attributed to a westward propagation of the initial arc intensification and correspondingly a duskward expansion of the arc-related current circuit. However, we also note that the $B_{\text {azi }}$ deviation on TH-D reduces earlier than that on TH-A/E. Referring to the optical observations shown in the top panel, such an earlier reduction is not owing to a concurrent drop of the auroral intensity along the TH-D meridian. Instead, the observation can be interpreted as follows: since TH-D is the outermost probe, it is the first to exit the transition region between the QPEB and AR, and enter deeply into the AR, where the radial closure current and its magnetic effect are supposed to be relaxed.

Combining the above observations and analyses, we conclude that the observed $B_{\text {azi }}$ perturbations on the three probes provide concrete support to the scenario of radially directed currents in the equatorial CPS as the closure of the arc-related FAC system, and further verify the probe geometry with respect to the QPEB and AR, carriers of the downward and upward FACs respectively, as shown in Fig. 13a.

We note, however, that there is a much stronger auroral brightening after $\sim$ 05:00 UTC, signifying substorm onset, but such a breakup is accompanied by virtually no $B_{\text {azi }}$ perturbations until the local dipolarization begins. Furthermore, as one can see from the TH-A observations in Fig. 9a, the QPEB features terminate right at the onset $\sim$ 05:00 UTC. It is well conceivable that the current system and its closure scheme would alter abruptly at the substorm onset; the downward "return" FAC related to the breakup arc could be remotely displaced, e.g., as in the scenario of a substorm current wedge, rather than located in the equatorward vicinity of the arc as in quiet and growth-phase intervals of this study's interest. Therefore, our proposed scenario (Fig. 2) and technique may not be applicable to a post-breakup arc.

We shall then check the relative geometry of the inferred AR location with respect to the NTR. Similar to Event 1, we see in Fig. 9 that the $B_{\mathrm{Z}}$ component first shows a steadily decreasing trend before $\sim 04: 45$ UTC, at a slope faster than $R^{-3}$, and then becomes relatively stable before the local dipolarization. Such a trend is more pronounced on TH-A than on TH-E because TH-A is closer to the neutral sheet. More specifically, the $B_{\mathrm{Z}}$ component on TH-A is initially larger than that on TH-E when they both show decreasing trend, yet the $B_{\mathrm{Z}}$ components on the two satellites become fairly close to each other $(\sim 6 \mathrm{nT})$ when they both reach a relatively stable level. These observations are consistent with the scenario that the two probes separated in the $\mathrm{z}$ direction both undergo a transition from a quasi-dipolar field to a stretched field topology. Such a transition is again a convolution of the outward motion of the probes and the inward migration of the NTR, the latter being a corollary of the gradual stretching of near-Earth CPS during the substorm growth phase. The geometry of TH-A and TH-E enables us to further estimate the radii of curvature of the equatorial magnetic field according to $R_{\mathrm{c}} \approx B_{\mathrm{Z}} \cdot\left(d B_{\text {rad }} / d z\right)^{-1}$ (Sergeev et al., 1983, 2012), in which $B_{\text {rad }}$ denotes the radial component of the B field. We use TH-A $B_{\mathrm{Z}}$ in the calculation due to its proximity to the neutral sheet, and estimate $d B_{\text {rad }} / d z$ from the two-probe measurements. The resulting $R_{\mathrm{c}}$ is shown in Fig. 13c. The estimation may be subject to uncertainty in the presence of the embedded thin current sheet between TH-E and TH-A, but the result can nevertheless serve as a rough indicator of the large-scale change of the overall magnetic field topology. Figure $13 \mathrm{c}$ reveals that $R_{\mathrm{c}}$ first steadily decreases until $\sim$ 04:45 UTC, and then becomes relatively stable until the local substorm dipolarization begins. A stable $R_{\mathrm{c}}$ value of $\sim 0.4 R_{\mathrm{E}}$ strongly hints that the probes encounter a highly stretched current sheet. The above behaviors of $B_{\mathrm{Z}}$ and $R_{\mathrm{c}}$ yield compelling clues of the passage of the NTR. According to the abrupt change of the trend of $B_{\mathrm{Z}}$ and $R_{\mathrm{c}}$, the inner edge of a highly stretched current sheet (labeled as the "core" of NTR) can be identified as $X \sim-10.0, R \sim 10.2 R_{\mathrm{E}}$, which is again found as inward of, yet very close to (within a fraction of $R_{\mathrm{E}}$ ), the earthward boundary of AR inferred from the above QPEB analyses. The result is thus fully consistent with that in Event 1.

The bottom panel of Fig. 13c shows the azimuthal component of the ion flows observed by TH-A and TH-E. Similar to Event 1, the flows contain both strong duskward DC components and ULF wave oscillations. The duskward flows peak around the core of the NTR and show an overall weak 
decreasing trend, albeit in an oscillating fashion, throughout the intensified QPEB region toward farther radial distances (see also Fig. 9), until the local dipolarization begins. The duskward flow peak appears earlier on the outer probe TH-E than on the inner probe TH-A, suggesting that the duskward flow enhancement is more a spatial effect relevant to the NTR than a temporal effect. The above flow variations with respect to the NTR and QPEB region are essentially consistent with those inferred from Event 1.

\section{Summary and conclusion}

Upgoing electron beams from the ionosphere and their magnetospheric counterpart, QPEB, have been known and reported on the basis of LEO and magnetospheric satellite measurements for decades. The QPEB is often well distinguishable in the near-equator CPS, and can serve as an ideal tracer of the downward FAC region. In this study, we investigate the QPEB features from both LEO and magnetospheric observations, with focus on their potential relationship to the arc. Based upon a preliminary survey of FAST data we infer a scenario common to all the events examined, that the upgoing electron beam is often found in the equatorward vicinity of an inverted-V arc. Such a scenario hints at a potential way to locate the AR in the CPS, namely using the tailward boundary of the QPEB to infer the earthward boundary of AR. We demonstrate the use of such technique in two THEMIS events. Our estimation of the AR location is further corroborated by the evaluation of the peak proton precipitation region, and particularly by the magnetic signatures of the current circuit formed by the QPEB and the arc. We emphasize again that we do not claim to present a fully established method to directly locate the AR in the CPS, which is still extremely difficult so far. Instead, we suggest a possible technique to indirectly estimate the AR location using currently available observations from CPS probes such as THEMIS. We also admit that the scenario shown in Fig. 2 may not be exclusive in terms of the current closure scheme of the arc. The result of our technique may thus be better described as "suggestive" rather than "definitive". Nevertheless, for the two THEMIS events we present, evidence from several observations leads us to believe that our technique is successful, and that our results can be carried out in future explorations of the AR and the arc generation mechanism in the CPS.

1. In both events, the AR boundary is shown to be located near a NTR - i.e., a transition region from a quasidipolar magnetosphere to a stretched current sheet at some point. Although the two events presented above are rather dissimilar in terms of the probe location, the geomagnetic conditions (nonsubstorm in Event 1 and substorm growth phase in Event 2), and the ambient magnetospheric status reflected in the large differences in the arc latitude as well as the radial location of the NTR itself, the relative position of the inferred AR with respect to the NTR is nearly identical in the two events: the AR itself is situated in a highly stretched current sheet region with a fairly stable $B_{\mathrm{Z}}$ and $R_{\mathrm{c}}$, but its earthward boundary is very close to a quasi-dipolar region characterized by much steepened $B_{\mathrm{Z}}$ and $R_{\mathrm{c}}$ gradients. Such consistency is unlikely to be fortuitous only. As a matter of fact, the above result is fully consistent with a number of existing observations and theoretical proposals that an equatorward-most arc likely maps to somewhere in the earthward portion of a stretched thin current sheet, on the verge of a transition into the quasidipolar inner magnetosphere (Lui and Burrows, 1978; Galperin et al., 1992; Yahnin et al., 1997; Galperin and Bosqued, 1999; Sergeev et al., 2012). In particular, using an event-adaptive magnetic field model (Kubyshkina et al., 2011) tuned by THEMIS, GOES, and NOAA observations, Sergeev et al. (2012) mapped the prebreakup arc to the CPS, and found that the AR was situated at the outer edge of a "magnetic wall" region with strong $B_{\mathrm{Z}}$ and $R_{\mathrm{c}}$ gradient. Though the methodological approach of Sergeev et al. (2012) and that presented here are fundamentally different and independent, our conclusions are fairly similar. Such consistency certainly gives credence to both the techniques of Sergeev et al. (2012) and our own.

2. In both events, we find that duskward flows roughly peak around the NTR, while throughout a main part of the QPEB region, there is in general a decreasing trend of duskward DC flows toward farther radial distance. The observed ion bulk flow contains the convective drift and the diamagnetic drift. However, since the region of interest is in the earthward-most portion of the stretched current sheet as depicted above, the cross-tail current density would tend to decay, which thereby implies a small diamagnetic drift. A quantitative estimation can be made in Event 2, when the TH-A/E geometry allows us to estimate the cross-tail current density using the differences between their radial magnetic field components. The cross-tail current density is calculated as $\sim 1-1.4 \mathrm{nAm}^{-2}$ when the probe traverses the NTR. The diamagnetic drift of ions, assuming that they are the carrier of the cross-tail current (note that this assumption yields the upper limit of the ion diamagnetic drift), is $\sim 18-24 \mathrm{~km} \mathrm{~s}^{-1}$ accordingly, which is substantially smaller than the observed magnitude of ion duskward flows. It is thus reasonable to state that, in this event, the observed ion duskward flows are mainly composed of convective drifts. The in situ observation that the duskward convective flow peaks around the NTR leads to the expectation that a westward ionospheric flow peak would be preferentially situated within the intense proton precipitation region, which basically corresponds to the ionospheric projection of the NTR (Donovan et al., 2012), moderately equatorward of the arc. 
Such a prediction is partly verified from a FAST survey on the E-field pattern surrounding the equatorward-most arc during quiet time and the substorm growth phase, recently performed by our colleagues (Jiang et al., 2013). Their preliminary results indeed reveal that a northward E-field peak is often found equatorward of the arc by a fraction of a degree in ILAT, and embedded within a region of intense proton precipitations. More quantitative investigations on the flow patterns surrounding the arc in both the ionosphere and magnetosphere, and a potential role of the flows in contributing to the arc-related current system, will be the subject of a future publication.

Acknowledgements. We are grateful to NASA for support of the THEMIS and FAST missions. We acknowledge J. McFadden, C. Carlson, D. Larson, and K.-H. Glassmeier for their work on THEMIS ESA, SST and FGM; and the FAST ESA.NORSTAR and CGSM MSP project is supported by CSA and NSERC.

Topical Editor L. Blomberg thanks two anonymous referees for their help in evaluating this paper.

\section{References}

Abel, G. A., Fazakerley, A. N., and Johnstone, A. D.: Simultaneous acceleration and pitch angle scattering of field-aligned electrons observed by the LEPA on CRRES, J. Geophys. Res., 107, 1416, doi:10.1029/2001JA005090, 2002a.

Abel, G. A., Fazakerley, A. N., and Johnstone, A. D.: Statistical distributions of field-aligned electron events in the near-equatorial magnetosphere observed by the Low Energy Plasma Analyzer on CRRES, J. Geophys. Res., 107, 1393, doi:10.1029/2001JA005073, 2002b.

Angelopoulos, V., Chapman, J. A., Mozer, F. S., Scudder, J. D., Russell, C. T., Tsuruda, K., Mukai, T., Hughes, T. J., and Yumoto, K.: Plasma sheet electromagnetic power generation and its dissipation along auroral field lines, J. Geophys. Res., 107, 1181, doi:10.1029/2001JA900136, 2002.

Auster, H. U., Glassmeier, K. H., Magnes, W., Aydogar, O., Baumjohann, W., Constantinescu, D., Fischer, D., Fornacon, K. H., Georgescu, E., Harvey, P., Hillenmaier, O., Kroth, R., Ludlam, M., Narita, Y., Nakamura, R., Okrafka, K., Plaschke, F., Richter, I., Schwarzl, H., Stoll, B., Valavanoglou, A., and Wiedemann, M.: The THEMIS fluxgate magnetometer, Space Sci. Rev., 141, 235-264, doi:10.1007/s11214-008-9365-9, 2008..

Boehm, M. H., Clemmons, J., Wahlund, J. E., Eriksson, A., Eliasson, L., Blomberg, L., Kintner, P., and Hofner, H.: Observations of an upward-directed electron beam with the perpendicular temperature of the cold ionosphere, J. Geophys. Res., 22, 21032106, 1995.

Bonnell, J. W., Mozer, F. S., Delory, G. T., Hull, A. J., Ergun, R. E., Cully, C. M., Angelopoulos, V., and Harvey, P. R.: The electric field instrument (EFI) for THEMIS, Space Sci. Rev., 141, 303341, doi:10.1007/s11214-008-9469-2, 2008.

Bostrom, R.: A model of the auroral electrojets, J. Geophys. Res., 69, 4983-4999, 1964.
Burch, J. L., Reiff, P. H., and Sugiura, M.: Upward electron beams measured by DE-1: A primary source of dayside region-1 Birkeland currents, Geophys. Res. Lett., 10, 753-756, 1983.

Carlson, C. W., McFadden, J. P., Ergun, R. E., Temerin, M., Peria, W., Mozer, F. S., Klumpar, D. M., Shelley, E. G., Peterson, W. K., Moebius, E., Elphic, R., Strangeway, R. E., Cattell, C. A., and Pfaff, R.: FAST observations in the downward auroral current region: Energetic upgoing electron beams, parallel potential drops, and ion heating, Geophys. Res. Lett., 25, 2017-2020, 1998.

Cran-McGreehin, A. P. and Wright, A. N.: Current-voltage relationship in downward field-aligned current region, J. Geophys. Res., 110, A10S10, doi:10.1029/2004JA010870, 2005.

Cully, C. M., Ergun, R. E., Stevens, K., Nammari, A., and Westfall, J.: The THEMIS digital fields board, Space Sci. Rev., 141, 343355, doi:10.1007/s11214-008-9417-1, 2008.

Donovan, E., Spanswick, E., Liang, J., Grant, J., Jackel, B., and Greffen, M.: Magnetospheric Dynamics and the Proton Aurora, in: Auroral Phenomenology and Magnetospheric Processes: Earth and Other Planets, Geophysical Monograph Series, 197, 365378, 2012.

Dubyagin, S. V., Sergeev, V. A., Carlson, C. W., Marple, S. R., Pulkkinen, T. I., and Yahnin, A. G.: Evidence of near-Earth breakup location, Geophys. Res. Lett., 30, 1282 , doi:10.1029/2002GL016569, 2003.

Elphic, R. C., Bonnell, J. W., Straneway, R. J., Kepko, L., Ergun, R. E., McFadden, J. P., Carlson, C. W., Peria, W., Catell, C. A., Klumpar, D., Shelley, E., Peterson, W., Moebius, E., Kistler, L., and Pfaff, R.: The auroral current circuit and field-aligned currents observed by FAST, Geophys. Res. Lett., 25, 2033-2036, doi:10.1029/98GL01158, 1998.

Elphic, R. C., Bonnell, J., Strangeway, R. J., Carlson, C. W., Temerin, M., McFadden, J. P., Ergun, R. E., and Peria, W.: FAST observations of upward accelerated electron beams and the downward field-aligned current region, Geophysical Monograph Series, Magnetospheric Current Systems, 118, 173-180, 2000.

Ergun, R. E., Andersson, L., Tao, J., Angelopoulos, V., Bonnell, J., McFadden, J. P., Larson, D. E., Eriksson, S., Johansson, T., Cully, C. M., Newman, D. N., Goldman, M. V., Roux, A., Le Contel, O., Glassmeier, K.-H., and Baumjohann, W.: Observations of double layers in Earth's plasma sheet, Phys. Rev. Lett., 102, 155002, doi:10.1103/PhysRevLett.102.155002, 2009.

Galperin, Y. I. and Bosqued, J. M.: Stationary magnetospheric convection on November 24, 1981. 1. A case study of "pressure gradient/minimum-B" auroral arc generation, Ann. Geophys., 17, 358-374, doi:10.1007/s00585-999-0358-0, 1999.

Galperin, Y. I., Volosevich, A. V., and Zelenyi, L. M.: Pressure gradient structures in the tail neutral sheet as "roots of the arcs" with some effects of stochasticity, Geophys. Res. Lett., 19, 21632166, doi:10.1029/92GL02178, 1992.

Gkioulidou, M., Wang, C.-P., and Lyons, L. R.: Effect of selfconsistent magnetic field on plasma sheet penetration to the inner magnetosphere: Rice convection model simulations combined with modified Dungey force-balanced magnetic field solver, J. Geophys. Res., 116, A12213, doi:10.1029/2011JA016810, 2011.

Gorney, D. J., Chiu, Y. T., and Croley Jr., D. R.: Trapping of Ion Conics by Downward Parallel Electric Fields, J. Geophys. Res., 90, 4205-4210, doi:10.1029/JA090iA05p04205, 1985.

Haerendel, G.: Auroral arcs as sites of magnetic stress release, J. Geophys. Res., 112, A09214, doi:10.1029/2007JA012378, 2007. 
Hultqvist, B., Lundin, R., Stasiewicz, K., Block, L., Lindqvist, P.A., Gustafsson, G., Koskinen, H., Bahnsen, A., Potemra, T. A., and Zanetti, L. J.: Simultaneous observation of upward moving field-aligned energetic electrons and ions on auroral zone field lines, J. Geophys. Res., 86, 9765-9776, 1988.

Jiang, F., Strangeway, R. J., Kivelson, M. G., Weygand, J. M., Walker, R. J., Khurana, K. K., Nishimura, T., Angelopoulos, V., and Donovan, E. F.: In-situ observations of the "preexisting auroral arc" by THEMIS All Sky Imagers and the FAST spacecraft, J. Geophys. Res., 117, A05211, doi:10.1029/2011JA017128, 2012.

Jiang, F., Kivelson, M. G., Strangeway, R., Liang, J., Khurana, K., Walker, R., Angelopoulos, V., Donovan, E., and Kubyshkina, M.: Ionospheric and magnetospheric flow shear associated with the preexisting auroral arc, J. Geophys. Res., in preparation, 2013.

Klumpar, D. M.: Statistical distributions of the auroral electron albedo in the magnetosphere: Auroral Plasma Dynamics, Geophys. Monogr. Ser., vol. 80, edited by: Lysak, R. L., pp. 163-171, AGU, Washington, D.C., 1993.

Klumpar, D. M., Quinn, J. M., and Shelley, E. G.: Counterstreaming electrons at the magnetic equator near $9 R_{\mathrm{E}}$, Geophys. Res. Lett., 15, 1295-1298, 1988.

Kubyshkina, M., Sergeev, V., Tsyganenko, N., Angelopoulos, V., Runov, A., Donovan, E., Singer, H., Auster, U., and Baumjohann, W.: Time-dependent magnetospheric configuration and breakup mapping during a substorm, J. Geophys. Res., 116, A00I27, doi:10.1029/2010JA015882, 2011.

Lessard, M. R., Lotko, W., LaBelle, J., Peria, W., Carlson, C. W., Creutzberg, F., and Wallis, D. D.: Ground and satellite observations of the evolution of growth phase auroral arcs, J. Geophys. Res., 112, A09304, doi:10.1029/2006JA011794, 2007.

Lin, C. S., Mauk, B., Parks, G. K., DeForest, S., and McIlwain, C. E.: Temperature Characteristics of Electron Beams and Ambient Particles, J. Geophys. Res., 84, 2651-2654, doi:10.1029/JA084iA06p02651, 1979.

Lui, A. T. Y. and Burrows, J. R.: On the Location of Auroral Arcs Near Substorm Onsets, J. Geophys. Res., 83, 3342-3348, doi:10.1029/JA083iA07p03342, 1978.

Marklund, G.: Auroral arc classification scheme based on the observed arc-associated electric field pattern, Planet. Space Sci., 2, 193-211, 1984.

Marklund, G.: Electric fields and plasma processes in the auroral downward current region, below, within, and above the acceleration region, Space Sci. Rev., 142, 1-21, doi:10.1007/s11214008-9373-9, 2009.

Marklund, G. and Karlsson, T.: Characteristics of the auroral particle acceleration in the upward and downward current regions, Phys. Chem. Earth, 26, 81-96, 2001.

Marklund, G. T., Karlsson, T., Figueiredo, S., Johansson, T., Lindqvist, P.-A., André, M., Buchert, S., Kistler, L. M., and Fazakerley, A.: Characteristics of quasi-static potential structures observed in the auroral return current region by Cluster, Nonlin. Processes Geophys., 11, 709-720, doi:10.5194/npg-11-7092004, 2004.

McFadden, J. P., Carlson, C. W., Larson, D.,Ludlam, M., Abiad, R., Elliott, B., Turin, P., Marckwordt, M., and Angelopoulos, V.: The THEMIS ESA plasma instrument and in-flight calibration, Space Sci. Rev., 141, 277-302, doi:10.1007/978-0-387-898209_13, 2008.
Ohtani, S., Wing, S., Newell, P. T., and Higuchi, T.: Locations of nightside precipitation boundaries relative to $\mathrm{R} 2$ and $\mathrm{R} 1$ currents, J. Geophys. Res., 115, A10233, doi:10.1029/2010JA015444, 2010.

Pfaff, R., Carlson, C., Watzin, J., Everett, D., and Gruner, T.: An overview of the fast auroral snapshot (FAST) satellite, Space Sci. Rev., 98, 1-32, 2001.

Rönnmark, K.: Electron acceleration in the auroral current circuit, Geo. Res. Lett., 26, 983-986, 1999.

Rönnmark, K.: Auroral current-voltage relation, J. Geophys. Res., 107, 1430, doi:10.1029/2002JA009294, 2002.

Rönnmark, K. and Hamrin, M.: Auroral Electron acceleration by Alfvén waves and electrostatic fields, J. Geophys. Res., 105, 25333-25344, doi:10.1029/2000JA900103, 2000.

Rothwell, P. L., Silevitch, M. B., Block, L. P., and Fälthammar, C.-G.: Prebreakup Arcs: A Comparison Between Theory and Experiment, J. Geophys. Res., 96, 13967-13975, doi:10.1029/91JA01268, 1991.

Roux, A., Le Contel, O., Coillot, C., Bouabdellah, A., De la Porte, B., Alison, D., Ruocco, S., and Vassal, M. C.: The search coil magnetometer for THEMIS, Space Sci. Rev., 141, 265-275, doi:10.1007/s11214-008-9455-8, 2008.

Roux, A., Robert, P., Le Contel, O., Angelopoulos, V., Auster, U., Bonnell, J., Cully, C. M., Ergun, R. E., and McFadden, J. P.: A mechanism for heating electrons in the magnetopause current layer and adjacent regions, Ann. Geophys., 29, 2305-2316, doi:10.5194/angeo-29-2305-2011, 2011.

Samson, J. C., Lyons, L. R., Newell, P. T., Creutzberg, F., and Xu, B.: Proton aurora and substorm intensifications, Geophys. Res. Lett., 19, 2167-2170, 1992.

Sergeev, V. A., Sazhina, E. M., Tsyganenko, N. A., Lundblad, J. A., and Soraas, F.: Pitch-angle scattering of energetic protons in the magnetotail current sheet as the dominant source of their isotropic precipitation into the nightside ionosphere, Planet. Space Sci., 31, 1147-1155, 1983.

Sergeev, V., Nishimura, Y., Kubyshkina, M., Angelopoulos, V., Nakamura, R., and Singer, H.: Magnetospheric location of the equatorward prebreakup arc, J. Geophys. Res., 117, A01212, doi:10.1029/2011JA017154, 2012.

Shiokawa, K., Baumjohann, W., and Paschmann, G.: Bi-directional electrons in the near-Earth plasma sheet, Ann. Geophys., 21, 1497-1507, doi:10.5194/angeo-21-1497-2003, 2003.

Temerin, M., and Carlson, C. W.: Current-voltage relationship in the downward auroral current region, Geophys. Res. Lett., 25, 2365-2368, 1998.

Teste, A., Fontaine, D., Sauvaud, J.-A., Maggiolo, R., Canu, P., and Fazakerley, A.: CLUSTER observations of electron outflowing beams carrying downward currents above the polar cap by northward IMF, Ann. Geophys., 25, 953-969, doi:10.5194/angeo-25953-2007, 2007.

Thorne, R. M., Ni, B., Tao, X., Horne, R. B., and Meredith, N. P.: Scattering by chorus waves as the dominant cause of diffuse auroral precipitation, Nature, 467, 943-946, 2010.

Wolf, R. A., Kumar, V., Toffoletto, F. R., Erickson, G. M., Savoie, A. M., Chen, C. X., and Lemon, C. L.: Estimating local plasma sheet PV ${ }^{5 / 3}$ from single-spacecraft measurements, J. Geophys. Res., 111, A12218, doi:10.1029/2006JA012010, 2006.

Wright, A. N., Owen, C. J., Chaston, C. C., and Dunlop, M. W.: Downward current electron beam observed by Cluster and FAST, 
J. Geophys. Res., 113, A06202, doi:10.1029/2007JA012643, 2008.

Wygant, J. R., Keiling, A., Cattell, C. A., Lysak, R. L., Temerin, M., Kletzing, C. A., Scudder, J. D., Mozer, F. S., Streltsov, V., Lotko, W., and Russell, C. T.: Evidence for Kinetic Alfven Waves and parallel electron energyization at $4-6 R_{\mathrm{E}}$ altitudes in the plasmasheet boundary layer, J. Geophys. Res., 107, 1201, doi:10.1029/2001JA900113, 2002.

Yahnin, A. G., Sergeev, V. A., Gvozdevsky, B. B., and Vennerstrøm, S.: Magnetospheric source region of discrete auroras inferred from their relationship with isotropy boundaries of energetic particles, Ann. Geophys., 15, 943-958, doi:10.1007/s00585-9970943-z, 1997.
Zaharia, S. G. and Wang, C. P.: Plasma pressure constraints on magnetic field structure in the substorm growth phase, GEM workshop, Sante Fe, 2011.

Zhang, Y., Matsumoto, M., and Omura, Y.: Linear and Nonlinear Interactions of an Electron Beam with Oblique Whistler and Electrostatic Waves in the Magnetosphere, J. Geophys. Res., 98, 21353-21363, 1993.

Zou, S., Lyons, L. R., Nicolls, M. J., Heinselman, C. J., and Mende, S. B.: Nightside ionospheric electrodynamics associated with substorms: PFISR and THEMIS ASI observations, J. Geophys. Res., 114, A12301, doi:10.1029/2009JA014259, 2009. 